JOURNAL OF THE AMERICAN MATHEMATICAL SOCIETY

Volume 20, Number 2, April 2007, Pages 573-602

S 0894-0347(06)00540-6

Article electronically published on July 14, 2006

\title{
ON THE RESTRICTION OF DELIGNE-LUSZTIG CHARACTERS
}

\author{
MARK REEDER
}

\section{INTRODUCTION}

This paper was motivated by the following restriction problem for representations of finite orthogonal groups.

Let $\mathfrak{F}$ be an algebraic closure of a finite field $\mathfrak{f}$ of cardinality $q$, a power of a prime $p>2$. Let $G=S O(V)$ be the special orthogonal group of a $(2 n+1)$-dimensional $\mathfrak{F}$-space $V$ with nondegenerate quadratic form $Q$. Assume $V$ and $Q$ are defined over $\mathfrak{f}$, and let $F$ denote the corresponding Frobenius endomorphisms of $V$ and $G$. Fix $v \in V^{F}$ with $Q(v) \neq 0$ and let $H$ be the stabilizer of $v$ in $G$.

Let $\pi \in \operatorname{Irr}\left(G^{F}\right), \sigma \in \operatorname{Irr}\left(H^{F}\right)$ be complex irreducible cuspidal representations of the respective groups $G^{F}$ and $H^{F}$ of $\mathfrak{f}$-rational points. The problem is to compute the multiplicity

$$
\langle\pi, \sigma\rangle_{H^{F}}=\operatorname{dim} \operatorname{Hom}_{H^{F}}(\pi, \sigma)
$$

of $\sigma$ in the restriction of $\pi$ to $H^{F}$.

Using unpublished work of Bernstein and Rallis (independently) on $p$-adic orthogonal groups, it can be shown that

$$
\langle\pi, \sigma\rangle_{H^{F}}=0 \quad \text { or } \quad 1 .
$$

In this paper, we compute $\langle\pi, \sigma\rangle_{H^{F}}$ exactly, when $\pi$ and $\sigma$ are irreducible cuspidal Deligne-Lusztig representations 8 . We do not rely on the above-mentioned work of Bernstein and Rallis. Our calculation follows from a qualitative study of restrictions of Deligne-Lusztig characters for general simple algebraic groups, to be described later in this introduction.

To state our multiplicity result for orthogonal groups, we first recall the inducing data. Let $T \subset G, S \subset H$ be $F$-stable anisotropic tori in $G$ and $H$. There are unique partitions $\lambda=\left(j^{\lambda j}\right), \mu=\left(j^{\mu_{j}}\right)$ of $n$ (here $\lambda_{j}, \mu_{j}$ are the number of parts equal to j) such that

$$
T^{F} \simeq \prod_{j}\left(\mathfrak{f}_{2 j}^{1}\right)^{\lambda_{j}}, \quad S^{F} \simeq \prod_{j}\left(\mathfrak{f}_{2 j}^{1}\right)^{\mu_{j}},
$$

where, for any $d>1, \mathfrak{f}_{d}=\mathfrak{F}^{F^{d}}$ is the extension of $\mathfrak{f}$ in $\mathfrak{F}$ of degree $d$, and $\mathfrak{f}_{2 j}^{1}$ is the kernel of the norm mapping $\mathfrak{f}_{2 j}^{\times} \rightarrow \mathfrak{f}_{j}^{\times}$. The number of parts $\sum_{j} \mu_{j}$ is even if $H$ is split, and odd if $H$ is nonsplit.

Received by the editors June 17, 2005.

2000 Mathematics Subject Classification. Primary 20C33.

The author was supported by NSF grant DMS-0207231.

(C)2006 American Mathematical Society Reverts to public domain 28 years from publication 
Let $\chi \in \operatorname{Irr}\left(T^{F}\right)$ and $\eta \in \operatorname{Irr}\left(S^{F}\right)$ be irreducible characters of $T^{F}$ and $S^{F}$ which are regular in the sense that $\chi$ and $\eta$ have trivial stabilizers in the respective Weyl groups $W_{G}(T)^{F}$ and $W_{H}(S)^{F}$. We may write

$$
\chi=\otimes_{j} \chi_{j}, \quad \eta=\otimes_{j} \eta_{j},
$$

where

$$
\chi_{j}=\chi_{j 1} \otimes \cdots \otimes \chi_{j \lambda_{j}} \in \operatorname{Irr}\left(\left(\mathfrak{f}_{2 j}^{1}\right)^{\lambda_{j}}\right),
$$

each $\chi_{j k}$ is a character of $\mathfrak{f}_{2 j}^{1}$, and likewise for $\eta$. Let $\Gamma_{2 j} \simeq \mathbb{Z} / 2 j \mathbb{Z}$ be the Galois group of $\mathfrak{f}_{2 j} / \mathfrak{f}$.

Definition 1.1. We say that $\chi$ and $\eta$ intertwine if $\eta_{j k^{\prime}}$ is a $\Gamma_{2 j}$-conjugate of $\chi_{j k}$ for some $1 \leq j \leq n, 1 \leq k \leq \lambda_{j}, 1 \leq k^{\prime} \leq \mu_{j}$.

Note that $\chi$ and $\eta$ can intertwine even if $T \not \subset S$. However, if $\lambda$ and $\eta$ have no common parts, that is, if $\lambda_{j} \mu_{j}=0$ for all $j$, then $\chi$ and $\eta$ do not intertwine.

By Deligne-Lusztig induction, we have virtual representations $R_{T, \chi}^{G}$ of $G^{F}$ and $R_{S, \eta}^{H}$ of $H^{F}$, respectively. By the regularity assumptions on $\chi$ and $\eta$, these are actually irreducible characters, up to sign. In fact, we have

$$
(-1)^{\mathrm{rk} G} R_{T, \chi}^{G} \in \operatorname{Irr}\left(G^{F}\right), \quad(-1)^{\mathrm{rk} H} R_{S, \eta}^{H} \in \operatorname{Irr}\left(H^{F}\right) .
$$

These two irreducible characters are cuspidal, since $T$ and $S$ are anisotropic. We prove:

Theorem 1.2. Let $T$ and $S$ be anisotropic $F$-stable maximal tori in $G$ and $H$, respectively, and let $\chi \in \operatorname{Irr}\left(T^{F}\right), \eta \in \operatorname{Irr}\left(S^{F}\right)$ be regular characters. Then

$$
(-1)^{\mathrm{rk} G+\mathrm{rk} H}\left\langle R_{T, \chi}^{G}, R_{S, \eta}^{H}\right\rangle_{H^{F}}= \begin{cases}0 & \text { if } \eta, \chi \text { intertwine } \\ 1 & \text { if } \eta, \chi \text { do not intertwine. }\end{cases}
$$

If $T$ and $S$ are arbitrary $F$-stable maximal tori, but $\chi$ and $\eta$ are still regular, then the multiplicity is either zero or a power of two; see (9.9) below.

The multiplicity result 1.2 is used in [12] to verify some cases of the conjectures of [11] describing restrictions from $p$-adic $\mathrm{SO}_{2 n+1}$ to $\mathrm{SO}_{2 n}$ in terms of symplectic local root numbers and the parameterization of depth-zero supercuspidal $L$-packets given in [7].

As already mentioned, Theorem 1.2 follows from a qualitative result, in a general setting, on multiplicities of Deligne-Lusztig representations.

Let $G$ be a a connected simple algebraic group defined over $\mathfrak{f}$, and let $H$ be a connected reductive f-subgroup of $G$. Fix $F$-stable maximal tori $T \subset G$ and $S \subset H$, along with arbitrary characters $\chi \in \operatorname{Irr}\left(T^{F}\right)$ and $\eta \in \operatorname{Irr}\left(S^{F}\right)$.

From this data Deligne and Lusztig [8] construct virtual characters $R_{T, \chi}^{G}$ and $R_{S, \eta}^{H}$ on $G^{F}$ and $H^{F}$, respectively. Let $\langle,\rangle_{H^{F}}$ be the canonical pairing on virtual characters of $H^{F}$. We are interested in the multiplicity

$$
\left\langle R_{T, \chi}^{G}, R_{S, \eta}^{H}\right\rangle_{H^{F}}
$$

where $R_{T, \chi}^{G}$ is viewed as a virtual character of $H^{F}$, by restriction.

Let $B$ and $B_{H}$ be Borel subgroups of $G$ and $H$, respectively, and let $\delta$ be the minimum codimension of a $B_{H}$-orbit in $G / B$. The invariant $\delta$ is called the complexity of the $H$-variety $G / B$. The theory of complexity was first studied for reductive groups over fields of characteristic zero (cf. 1] and the references therein). In that 
setting, it is proved in [1] that $\delta$ governs the growth of multiplicities in restrictions of algebraic representations. We will show that $\delta$ also governs the growth of multiplicities in restrictions of Deligne-Lusztig representations.

Because we are in nonzero characteristic, we need to make an assumption. Let $\mathfrak{g}, \mathfrak{h}$ be the Lie algebras of $G$ and $H$.

Assumption 1.3. There is an $\operatorname{Ad}(H)$-stable decomposition $\mathfrak{g}=\mathfrak{h} \oplus \mathfrak{m}$, and a nondegenerate symmetric bilinear form $B$ on $\mathfrak{m}$, invariant under $\operatorname{Ad}(H)$.

This assumption holds if $p$ is a good prime for $\mathfrak{g}$ and the Killing form of $\mathfrak{g}$ is nondegenerate on $\mathfrak{h}$ [24, I.5.3]. For $G=S O_{N+1}, H=S O_{N}$, our assumption holds for $p>2$.

For an integer $\nu \geq 1$, let $N_{\nu}^{T}: T^{F^{\nu}} \rightarrow T^{F}$ be the norm map, and let

$$
\chi^{(\nu)}=\chi \circ N_{\nu}^{T}, \quad \eta^{(\nu)}=\eta \circ N_{\nu}^{S} .
$$

Under Assumption 1.3 we prove the following.

Theorem 1.4. There is a polynomial of degree at most $\delta$ :

$$
M(t)=A t^{\delta}+\cdots \in \mathbb{Q}[t],
$$

whose coefficients depend on $\chi$ and $\eta$, and an integer $m \geq 1$ such that

$$
\left\langle R_{T, \chi^{(\nu)}}^{G}, R_{S, \eta^{(\nu)}}^{H}\right\rangle_{H^{F^{\nu}}}=M\left(q^{\nu}\right)
$$

for all positive integers $\nu \equiv 1 \bmod m$. The degree $\delta$ is optimal: if $q$ is sufficiently large, there exist $\chi, \eta$ such that the leading coefficient $A$ is nonzero.

We also give an explicit formula for the leading term $A$ in Theorem 1.4 (see Proposition (7.4). For $G=S O_{N+1}, H=S O_{N}$, we have $\delta=0$, and our explicit formula for $A$ leads to Theorem 1.2 (see Section 9). Even if $\delta>0$ one can sometimes use Theorem 1.4 to compute exact multiplicities, by exploiting the polynomial nature of $M(t)$. In Section 10 we illustrate this for $G=S O_{7}, H=G_{2}$, where $\delta=1$.

Our formula for $A$ also allows us to show, for general $G$ and $H$, and "very regular" $\chi$ (see section 8), that the multiplicity

$$
\left\langle R_{T, \chi}^{G}, S t_{H}\right\rangle_{H^{F}}
$$

of the Steinberg representation $S t_{H}$ is a monic polynomial in $q$ of degree $\delta$, while the multiplicity of the trivial representation

$$
\left\langle R_{T, \chi}^{G}, 1_{H}\right\rangle_{H^{F}}
$$

is a polynomial in $q$ of degree strictly less than $\delta$. In particular, for $G=S O_{2 n+1}$ and $H=S O_{2 n}$, we have

$$
\left\langle R_{T, \chi}^{G}, S t_{H}\right\rangle_{H^{F}}=1, \quad\left\langle R_{T, \chi}^{G}, 1_{H}\right\rangle_{H^{F}}=0,
$$

for very regular $\chi$.

To prove Theorem 1.4 we use a method introduced by Thoma 27. for the study of the restriction of irreducible representations from $G L_{n}(\mathfrak{f})$ to $G L_{n-1}(\mathfrak{f})$ (where again $\delta=0$ ). In that situation, the Green's functions giving the character on unipotent elements were explicitly known. Hagedorn [13, in his 1994 Ph.D. thesis, showed how some of Thoma's methods could be generalized to Deligne-Lusztig characters for other pairs of classical groups, where the Green's functions are less explicit. The 
abstract results of Hagedorn gave me the courage to attempt such calculations for general groups and to obtain closed multiplicity formulas for orthogonal groups.

It is a pleasure to thank Dick Gross for initiating the work in [12] which led to this paper, for helpful remarks on an earlier version, and for aquainting me with Hagedorn's thesis.

The referee read the original version of this paper with care and insight, made valuable comments and simplified some of the arguments. In particular, the proof of Lemma 3.1 given below is due to the referee and is much shorter than the original one.

Some general notation: The cardinality of a finite set $X$ is denoted by $|X|$. Equivalence classes are generally denoted by $[\cdot]$, sometimes with ornamentation. If $g$ is an element of a group $G$, we write $\operatorname{Ad}(g)$ for the conjugation map $\operatorname{Ad}(g)$ : $x \mapsto g x g^{-1}$, and also write ${ }^{g} T:=g T g^{-1}$ for a subgroup $T \subset G$. The center of $G$ is denoted $Z(G)$ and the centralizer of $g \in G$ is denoted $C_{G}(g)$.

We write $\langle,\rangle_{H}$ for the pairing on the space of class functions on a finite group $H$, for which the irreducible characters of $H$ are an orthonormal basis. If $G, G^{\prime} \supset H$ are finite overgroups of $H$ and $\psi, \psi^{\prime}$ are class functions on $G, G^{\prime}$ respectively, then $\left\langle\psi, \psi^{\prime}\right\rangle_{H}$ is understood to mean $\left\langle\left.\psi\right|_{H},\left.\psi^{\prime}\right|_{H}\right\rangle_{H}$, where $\left.\right|_{H}$ denotes restriction to $H$.

\section{REMARKS ON MAXIMAL TORI}

Let $G$ be a connected reductive algebraic $\mathfrak{F}$-group. We assume $G$ is defined over $\mathfrak{f}$ and has Frobenius $F$. If $T$ is a maximal torus in $G$ we denote its normalizer in $G$ by $N_{G}(T)$ and write $W_{G}(T)=N_{G}(T) / T$ for the Weyl group of $T$ in $G$. If $T$ is $F$-stable, we have

$$
W(T)^{F}=N_{G}(T)^{F} / T^{F}
$$

by the Lang-Steinberg theorem.

The reduction formula for Deligne-Lusztig characters (recalled in section 4 below) involves a sum over the following kind of subset of $G^{F}$. Fix an $F$-stable maximal torus $T \subset G$, and let $s$ be a semisimple element in $G^{F}$. We must sum over the set

$$
N_{G}(s, T)^{F}:=\left\{\gamma \in G^{F}: s^{\gamma} \in T\right\} .
$$

Note that $N_{G}(s, T)^{F}$, if nonempty, is a union of $G_{s}^{F} \times N_{G}(T)^{F}$ double cosets, where $G_{s}:=C_{G}(s)^{\circ}$ is the identity component of the centralizer $C_{G}(s)$ of $s$ in $G$.

To say that $s^{\gamma} \in T$ is to say that ${ }^{\gamma} T \subset G_{s}$, so determining the $G_{s}^{F} \times N_{G}(T)^{F}$ double cosets in $N_{G}(s, T)^{F}$ amounts to determining the $G_{s}^{F}$-conjugacy classes of $F$-stable maximal tori in $G_{s}$ which are contained in a given $G^{F}$-conjugacy class. Such classes of tori are parameterized by twisted conjugacy classes in Weyl groups of $G_{s}$ and $G$.

The aim of this section is to parameterize the $G_{s}^{F} \times N_{G}(T)^{F}$ double cosets in $N_{G}(s, T)^{F}$ in terms of the fiber of a natural map between twisted conjugacy classes in the Weyl groups of $G_{s}$ and $G$. This parameterization will be fundamental to our later calculations with Deligne-Lusztig characters.

We begin by recalling the classification of $F$-stable maximal tori in $G$. See [5, chap. 3] for more details in what follows. Fix an $F$-stable maximal torus $T_{0}$ in $G$ contained in an $F$-stable Borel subgroup of $G$, and abbreviate $N_{G}=N_{G}\left(T_{0}\right)$, $W_{G}=W_{G}\left(T_{0}\right)$. 
Let $\mathcal{T}(G)$ denote the set of all $F$-stable maximal tori in $G$. Then $\mathcal{T}(G)$ is a finite union of $G^{F}$-orbits. For any $T \in \mathcal{T}(G)$, let

$$
[T]_{G}:=\left\{{ }^{\gamma} T: \gamma \in G^{F}\right\}
$$

denote the $G^{F}$-orbit of $T$. There is $g \in G$ such that $T={ }^{g} T_{0}$. Since $T$ is $F$-stable, we have $g^{-1} F(g) \in N_{G}$. This gives an element

$$
w:=g^{-1} F(g) T_{0} \in W_{G} .
$$

The map $\operatorname{Ad}(g) t=g t g^{-1}$ is an $\mathfrak{f}$-isomorphism

$$
\operatorname{Ad}(g):\left(T_{0}, w F\right) \longrightarrow(T, F),
$$

where the second component denotes the action of Frobenius under an $\mathfrak{f}$-structure.

For any finite group $A$ with $F$-action, we let $H^{1}(F, A)$ denote the set of $F$ conjugacy classes in $A$. These are the orbits of the action of $A$ on itself via $(a, b) \mapsto$ $a b F(a)^{-1}$. Let $[b] \in H^{1}(F, A)$ denote the $F$-conjugacy class of an element $b \in A$.

For $g, T, w$ as above, the $F$-conjugacy class of $w$ is independent of the choice of $g$. Hence we have a well-defined class

$$
\operatorname{cl}(T, G):=[w] \in H^{1}\left(F, W_{G}\right) .
$$

For each $\omega \in H^{1}\left(F, W_{G}\right)$, the set

$$
\mathcal{T}_{\omega}(G):=\{T \in \mathcal{T}(G): \operatorname{cl}(T, G)=\omega\}
$$

is a single $G^{F}$-orbit in $\mathcal{T}(G)$, and all $G^{F}$-orbits are of this form. Thus, the partition of the set of $F$-stable maximal tori into $G^{F}$-orbits is given by

$$
\mathcal{T}(G)=\coprod_{\omega \in H^{1}\left(F, W_{G}\right)} \mathcal{T}_{\omega}(G) .
$$

Let $s \in G^{F}$ be semisimple, and let $T_{s}$ be an $F$-stable maximal torus of $G_{s}$ contained in an $F$-stable Borel subgroup of $G_{s}$, and let $W_{G_{s}}$ be the Weyl group of $T_{s}$ in $G_{s}$. The partition of $\mathcal{T}\left(G_{s}\right)$ into $G_{s}^{F}$-orbits is given, as above, by

$$
\mathcal{T}\left(G_{s}\right)=\coprod_{v \in H^{1}\left(F, W_{G_{s}}\right)} \mathcal{T}_{v}\left(G_{s}\right)
$$

If $T \in \mathcal{T}(G)$, the set of $F$-stable maximal tori in $G_{s}$ which are $G^{F}$-conjugate to $T$ is a finite union (possibly empty) of $G_{s}^{F}$-orbits. We want to describe this union in terms of $F$-conjugacy classes in $W_{G_{s}}$. That is, given $\omega \in H^{1}\left(F, W_{G}\right)$, we have

$$
\mathcal{T}_{\omega}(G) \cap \mathcal{T}\left(G_{s}\right)=\coprod_{v \in M_{\omega}} \mathcal{T}_{v}\left(G_{s}\right)
$$

for some subset $M_{\omega} \subseteq H^{1}\left(F, W_{G_{s}}\right)$, and our task is to find $M_{\omega}$.

The first point is that $T_{s}$ is generally not contained in an $F$-stable Borel subgroup of $G$. Let $g \in G$ be such that ${ }^{g} T_{s}=T_{0}$, and let $\dot{y}_{s}:=g F(g)^{-1}$ have image $y_{s} \in W_{G}$. Then

and $\operatorname{Ad}(g)$ is an $\mathfrak{f}$-isomorphism

$$
\operatorname{cl}\left(T_{s}, G\right)=\left[y_{s}\right] \in H^{1}\left(F, W_{G}\right),
$$

$$
\operatorname{Ad}(g):\left(T_{s}, F\right) \longrightarrow\left(T_{0}, y_{s} F\right) .
$$

Now $T_{0}$ is also a maximal torus in $\operatorname{Ad}(g) G_{s}$, whose Weyl group

$$
W_{G_{s}}^{\prime}:=\operatorname{Ad}(g) W_{G_{s}}
$$

is a subgroup of $W_{G}$, stable under $\operatorname{Ad}\left(y_{s}\right) \circ F$. 
Define $j_{G_{s}}: H^{1}\left(F, W_{G_{s}}\right) \rightarrow H^{1}\left(F, W_{G}\right)$ to be the composition of maps

$$
j_{G_{s}}: H^{1}\left(F, W_{G_{s}}\right) \stackrel{\operatorname{Ad}(g)}{\longrightarrow} H^{1}\left(y_{s} F, W_{G_{s}}^{\prime}\right) \stackrel{\text { incl }}{\longrightarrow} H^{1}\left(y_{s} F, W_{G}\right) \stackrel{\tau_{y_{s}}}{\longrightarrow} H^{1}\left(F, W_{G}\right),
$$

where the middle map is induced by the inclusion $W_{G_{s}}^{\prime} \hookrightarrow W_{G}$ and $\tau_{y_{s}}$ is the twisting bijection given by $\tau_{y_{s}}[x]=\left[x y_{s}\right]$.

Now let $T$ be an arbitrary $F$-stable maximal torus in $G_{s}$. Write $T={ }^{h} T_{s}$, with $h \in G_{s}$, so that $h^{-1} F(h) \in \operatorname{cl}\left(T, G_{s}\right)$. For $g \in G$ as above, we have $T=h g^{-1} T_{0}$. Since

$$
g h^{-1} F\left(h g^{-1}\right)=g\left(h^{-1} F(h)\right) g^{-1} \cdot g F(g)^{-1},
$$

it follows that

$$
\operatorname{cl}(T, G)=j_{G_{s}}\left(\operatorname{cl}\left(T, G_{s}\right)\right) .
$$

This proves:

Lemma 2.1. For each $\omega \in H^{1}\left(F, W_{G}\right)$ and $T \in \mathcal{T}_{\omega}\left(G_{s}\right)$, we have

$$
\mathcal{T}_{\omega}(G) \cap \mathcal{T}\left(G_{s}\right)=\coprod_{v \in j_{G_{s}}^{-1}(\omega)} \mathcal{T}_{v}\left(G_{s}\right) .
$$

We can also parameterize the $G_{s}^{F}$-orbits in $[T]_{G} \cap \mathcal{T}\left(G_{s}\right)$ via the mapping

$$
N_{G}(s, T)^{F}:=\left\{\gamma \in G^{F}: s \in{ }^{\gamma} T\right\} \longrightarrow[T]_{G} \cap \mathcal{T}\left(G_{s}\right), \quad \gamma \mapsto^{\gamma} T .
$$

Note that $G_{s}^{F}$ acts on $N_{G}(s, T)^{F}$ by left multiplication, and that (2.4) factors through the quotient

$$
\bar{N}_{G}(s, T)^{F}:=G_{s}^{F} \backslash N_{G}(s, T)^{F} .
$$

The action of $N_{G}(T)^{F}$ on $N_{G}(s, T)^{F}$ by right multiplication commutes with the $G_{s}^{F}$-action, hence factors through an action on $\bar{N}_{G}(s, T)^{F}$, where $T^{F}$ acts trivially. This gives an action of $W_{G}(T)^{F}$ on $\bar{N}_{G}(s, T)^{F}$, whose orbits are the $G_{s}^{F} \times N(T)^{F}$ double cosets in $N(s, T)^{F}$.

Lemma 2.2. The mapping (2.4), sending $\gamma \mapsto{ }^{\gamma} T$, induces a bijection

$$
\bar{N}_{G}(s, T)^{F} / W_{G}(T)^{F} \stackrel{\sim}{\longrightarrow} G_{s}^{F} \backslash\left([T]_{G} \cap \mathcal{T}\left(G_{s}\right)\right)
$$

with the property that the stabilizer in $W_{G}(T)^{F}$ of the class $\bar{\gamma} \in \bar{N}(s, T)^{F}$ is isomorphic, via $\operatorname{Ad}(\gamma)$, to $W_{G_{s}}\left({ }^{\gamma} T\right)^{F}$.

Proof. The bijectivity is straightforward and left to the reader. Let $w \in W_{G}(T)^{F}$, and let $\dot{w} \in N_{G}(T)^{F}$ be a representative of $w$. Then

$$
\bar{\gamma} \cdot w=\bar{\gamma} \quad \Leftrightarrow \quad G_{s}^{F} \gamma \dot{w}=G_{s}^{F} \gamma \quad \Leftrightarrow \quad \operatorname{Ad}(\gamma) \dot{w} \in N_{G_{s}}\left({ }^{\gamma} T\right) .
$$

This implies the assertion about the stabilizer.

Combining Lemmas 2.1 and 2.2, we get an explicit formula for $\left|\bar{N}_{G}(s, T)^{F}\right|$.

Corollary 2.3. Let $\omega \in H^{1}\left(F, W_{G}\right)$ and $T \in \mathcal{T}_{\omega}(G)$. Then the set $N_{G}(s, T)^{F}$ is nonempty if and only if the fiber $j_{G_{s}}^{-1}(\omega)$ is nonempty, in which case we have

$$
\left|\bar{N}_{G}(s, T)^{F}\right|=\sum_{v \in j_{G_{s}}^{-1}(\omega)} \frac{\left|W_{G}(T)^{F}\right|}{\left|W_{G_{s}}\left(T_{v}\right)^{F}\right|},
$$

where, for each $v \in j_{G_{s}}^{-1}(\omega)$, the torus $T_{v}$ is chosen arbitrarily in $\mathcal{T}_{v}\left(G_{s}\right)$. 


\section{ON THE CENTRALIZER OF A SEMISIMPLE ELEMENT}

Let $s \in G^{F}$ be semisimple. In the previous section we parameterized the set of $G_{s}^{F}$-conjugacy classes of maximal tori in $G_{s}$ which are contained in a given $G^{F}$ conjugacy class, in terms of fibers of the map $j_{G_{s}}: H^{1}\left(F, W_{G_{s}}\right) \rightarrow H^{1}\left(F, W_{G}\right)$. To compute this map $j_{G_{s}}$ concretely, we must find an element $y_{s} \in W_{G}$ such that $\operatorname{cl}\left(T_{s}, G\right)=\left[y_{s}\right]$, where $T_{s} \in \mathcal{T}\left(G_{s}\right)$ is contained in an $F$-stable Borel subgroup of $G_{s}$. This amounts to finding the f-isomorphism class of the connected centralizer $G_{s}$.

An elegant formula for $y_{s}$ was given by Carter [6], using the Brauer complex. Here we explain a different method that is suited to our later computations; namely we show how the class $\left[y_{s}\right]$ can be determined from the effect of $F$ on a "diagonalized" $G$-conjugate of $s$. Unfortunately, both the present method, as well as that of [6] require that $C_{G}(s)$ be connected. That is, we must assume that $G_{s}=C_{G}(s)$. This holds for any semisimple $s \in G$ if $G$ has simply-connected derived group. Our method generalizes that of Gross [10, who determined $C_{G}(s)$ when this group is a torus (over an arbitrary field).

Let $\Phi$ denote the set of roots of $T_{0}$ in $G$. Let $\vartheta$ denote the automorphisms of $\Phi$ and $W_{G}$ induced by $F$. For $\alpha \in \Phi$ with corresponding reflection $s_{\alpha} \in W_{G}$, we have

$$
\alpha \circ F=q \vartheta^{-1} \cdot \alpha, \quad \vartheta\left(s_{\alpha}\right)=s_{\vartheta} \cdot \alpha
$$

Here is our recipe for finding $\operatorname{cl}\left(T_{s}, G\right)$. Let $t \in T_{0}$ be a $G$-conjugate of $s$, and let

$$
\Phi_{t}=\{\alpha \in \Phi: \alpha(t)=1\} .
$$

Since $t$ has a conjugate in $G^{F}$, there is $w \in W$ (not necessarily unique) such that

$$
F(t)=t^{w} \text {. }
$$

Choose such a $w$ arbitrarily. From (3.1) it follows that

$$
w \vartheta \cdot \Phi_{t}=\Phi_{t} .
$$

Now choose any positive system $\Phi_{t}^{+} \subset \Phi_{t}$. Then (3.2) implies that $w \vartheta \cdot \Phi_{t}^{+}$is another positive system in $\Phi_{t}$. Being the Weyl group of $\Phi_{t}$, the group $W_{G_{t}}$ acts simply transitively on positive systems in $\Phi_{t}$, so there is a unique $x \in W_{G_{t}}$ such that

$$
w \vartheta \cdot \Phi_{t}^{+}=x \cdot \Phi_{t}^{+} .
$$

Setting $y=x^{-1} w$, we see that $w$ can be factored uniquely as

$$
w=x y,
$$

where $x \in W_{G_{t}}$ and $y \vartheta \cdot \Phi_{t}^{+}=\Phi_{t}^{+}$.

Since $C_{G}(t)$ is connected, the group $W_{G_{t}}$ is the full stabilizer of $t$ in $W_{G}$. This means that a different choice of $w$ satisfying (3.1) will change $x$, but not $y$.

Lemma 3.1. With y constructed as above, we have

$$
\operatorname{cl}\left(T_{s}, G\right)=[y] \in H^{1}\left(F, W_{G}\right) .
$$

Proof. The following proof was provided by the referee; it is shorter than the original proof. Choose $g \in G$ such that $\dot{y}=g^{-1} F(g) \in N_{G}$ is a representative of $y$. Then

$$
y F(t)=t^{x}=t,
$$


which implies that ${ }^{g} t \in G^{F}$. Since $C_{G}(s)$ is connected, any element of $G^{F}$ which is $G$-conjugate to $s$ is in fact $G^{F}$-conjugate to $s$. Hence, by multiplying $g$ on the left by an element of $G^{F}$, we may assume that $s={ }^{g} t$.

By definition of $y$, there is an $\operatorname{Ad}(\dot{y}) F$-stable Borel subgroup $B_{t} \subset G_{t}$ containing $T_{0}$. Hence ${ }^{g} B_{t}$ is an $F$-stable Borel subgroup of $G_{s}$, containing the $F$-stable maximal torus $T_{s}^{\prime}:={ }^{g} T_{0}$. Since $T_{s}^{\prime}$ is $G_{s}^{F}$-conjugate to $T_{s}$, it follows that

$$
\operatorname{cl}\left(T_{s}, G\right)=\left[g^{-1} F(g)\right]=[y],
$$

as claimed.

\section{Deligne-Lusztig CharaCters}

Let $T \in \mathcal{T}(G)$ be an $F$-stable maximal torus in $G$, and let $\chi \in \operatorname{Irr}\left(T^{F}\right)$. The Deligne-Lusztig character $R_{T, \chi}^{G}$ has the following reduction formula [8]: For $u$ unipotent in $G_{s}^{F}$, we have

$$
R_{T, \chi}^{G}(s u)=\sum_{\bar{\gamma} \in \bar{N}_{G}(s, T)^{F}} \chi\left(\gamma^{-1} s \gamma\right) Q_{\gamma T \gamma^{-1}}^{G_{s}}(u) .
$$

The summation is over the set $\bar{N}_{G}(s, T)^{F}$ defined in (2.5), and for any reductive f-group $H$, and $S \in \mathcal{T}(H)$, the Green function $Q_{S}^{H}$ on the unipotent set of $H^{F}$ is defined by

$$
Q_{S}^{H}(u)=R_{S, 1}^{H}(u) .
$$

In this section we describe the summation over $\bar{N}_{G}(s, T)^{F}$ in (4.1) in terms of fibers of the map $j_{G_{s}}$ studied in the previous two sections.

Breaking the sum (4.1) into $W_{G}(T)^{F}$-orbits, we have

$$
R_{T, \chi}^{G}(s u)=\sum_{v \in j_{G_{s}}^{-1}(\omega)} Q_{T_{v}}^{G_{s}}(u) \sum_{\bar{\gamma} \in \mathcal{O}_{v}} \chi\left(\gamma^{-1} s \gamma\right),
$$

where $\omega=\operatorname{cl}(T, G), T_{v}$ is any torus in $\mathcal{T}_{v}\left(G_{s}\right)$, and $\mathcal{O}_{v}$ is the $W_{G}(T)^{F}$-orbit in $\bar{N}_{G}(s, T)^{F}$ corresponding to $v \in j_{G_{s}}^{-1}(\omega)$ as in Lemma 2.2

By the stabilizer assertion in Lemma 2.2, the inner sum in (4.2) can be written as follows. For any $\gamma \in N_{G}(s, T)^{F}$ and $\chi^{\prime} \in \operatorname{Irr}\left(T^{F}\right)$, the value at $s$ of the transported character

$$
{ }^{\gamma} \chi^{\prime}:=\chi^{\prime} \circ \operatorname{Ad}\left(\gamma^{-1}\right) \in \operatorname{Irr}\left({ }^{\gamma} T^{F}\right)
$$

depends only on the image $\bar{\gamma} \in \bar{N}_{G}(s, T)^{F}$. We have

$$
\sum_{\bar{\gamma} \in \mathcal{O}_{v}} \chi\left(\gamma^{-1} s \gamma\right)=\frac{1}{\left|W_{G_{s}}\left(T_{v}\right)^{F}\right|} \sum_{x \in W_{G}(T)^{F}} \gamma x \chi(s),
$$

where $\gamma$ on the right side of (4.3) is an arbitrary element of $N_{G}(s, T)^{F}$ such that $\bar{\gamma} \in \mathcal{O}_{v}$.

In our later computations with $R_{T, \chi}^{G}$ it will be useful to let $s$ vary in $G^{F}$ in such a way that $G_{s}$ is unchanged. Let $Z\left(G_{s}\right)$ denote the center of $G_{s}$. For $v \in H^{1}\left(F, W_{G_{s}}\right)$, the function

$$
\chi_{v}:=\sum_{\bar{\gamma} \in \mathcal{O}_{v}}{ }^{\gamma} \chi
$$


is well-defined on $Z\left(G_{s}\right)^{F}$, and we have

$$
R_{T, \chi}^{G}(z u)=\sum_{v \in j_{G_{s}}^{-1}(\omega)} Q_{T_{v}}^{G_{s}}(u) \chi_{v}(z), \quad \text { if } G_{z}=G_{s} .
$$

\section{Multiplicity AS A POLYNOMial}

In this section we begin the proof of Theorem 1.4 and will show that the multiplicity is given by a polynomial function. Let $G$ be a connected reductive algebraic group over $\mathfrak{f}$. Let $H \subset G$ be a connected reductive $\mathfrak{f}$-subgroup of $G$, and let $S$ be an $F$-stable maximal torus of $H$.

5.1. Summation on $H^{F}$. Suppose we are given a function $f: H^{F} \rightarrow \mathbb{C}$, invariant under conjugation by $H^{F}$, with the property that if $h \in H^{F}$ has Jordan decomposition $h=s u$, then $f(h)=0$ unless the conjugacy class $\operatorname{Ad}\left(H^{F}\right) \cdot s$ meets $S$. Our first aim is to express the sum of $f$ over $H^{F}$ as a sum of rational functions in $q$ over an index set which does not depend on $q$.

Let $H^{\text {ss }}$ and $H^{\text {upt }}$ be the sets of semisimple and unipotent elements of $H$. Let $\mathcal{S}\left(H^{F}\right)$ and $\mathcal{U}\left(H^{F}\right)$ be the sets of $\operatorname{Ad}\left(H^{F}\right)$-orbits in $\left(H^{\text {ss }}\right)^{F}$ and $\left(H^{\text {upt }}\right)^{F}$, respectively.

By the vanishing assumption on $f$, we have

$$
\begin{aligned}
\frac{1}{\left|H^{F}\right|} \sum_{h \in H^{F}} f(h) & =\frac{1}{\left|H^{F}\right|} \sum_{s \in\left(H^{\mathrm{ss}}\right)^{F}} \sum_{u \in\left(H_{s}^{\mathrm{upt}}\right)^{F}} f(s u) \\
& =\frac{1}{\left|H^{F}\right|} \sum_{s \in S^{F}} \frac{\left|\operatorname{Ad}\left(H^{F}\right) \cdot s\right|}{\left|\operatorname{Ad}\left(H^{F}\right) \cdot s \cap S\right|} \sum_{[u] \in \mathcal{U}\left(H_{s}^{F}\right)}\left|\operatorname{Ad}\left(H_{s}^{F}\right) \cdot u\right| f(s u) .
\end{aligned}
$$

The map $\gamma \mapsto s^{\gamma}$ induces a bijection

$$
C_{H}(s)^{F} \backslash N_{H}(s, S)^{F} \stackrel{\sim}{\longrightarrow} \operatorname{Ad}\left(H^{F}\right) \cdot s \cap S,
$$

so that

Recalling that

$$
\left|\operatorname{Ad}\left(H^{F}\right) \cdot s \cap S\right|=\frac{\left|N_{H}(s, S)^{F}\right|}{\left|C_{H}(s)^{F}\right|}
$$

$$
\bar{N}_{H}(s, S)^{F}=H_{s}^{F} \backslash N_{H}(s, S)^{F}
$$

we get

$$
\frac{1}{\left|H^{F}\right|} \sum_{h \in H^{F}} f(h)=\sum_{s \in S^{F}} \frac{1}{\left|\bar{N}_{H}(s, S)^{F}\right|} \sum_{[u] \in \mathcal{U}\left(H_{s}^{F}\right)} \frac{1}{\left|C_{H_{s}}(u)^{F}\right|} f(s u) .
$$

5.2. A partition of $S$. To this point, the overgroup $G$ has not played a role. Now $G$ is used to partition the sum over $S^{F}$ in (5.2), as follows. Let $I(S)$ be an index set for the set of subgroups

$$
\left\{G_{s}: s \in S\right\} \text {. }
$$

Note that each element of $I(S)$ is determined by a subset of the roots of $S$ in $G$; hence $I(S)$ is finite. For $\iota \in I(S)$ let $G_{\iota}$ be the corresponding connected centralizer, and let

$$
S_{\iota}:=\left\{s \in S: G_{s}=G_{\iota}\right\}
$$


Thus, $S$ is finitely partitioned as

$$
S=\coprod_{\iota \in I(S)} S_{\iota}
$$

The $F$-action on $S$ induces a permutation of $I(S)$, and we let $I(S)^{F}$ be the $F$-fixed points in $I(S)$. Note that if $S_{\iota}^{F}$ is nonempty, then $\iota \in I(S)^{F}$.

For $\iota \in I(S)$, we set

$$
H_{\iota}:=\left(H \cap G_{\iota}\right)^{\circ},
$$

which is none other than $H_{s}$ for any $s \in S_{\iota}$.

Note that if $s \in S_{\iota}$, then $s \in S \cap C_{H}(s)$, which implies that

$$
s \in H_{\iota} \subset G_{\iota} .
$$

Returning to our sum (5.2), we now have

$$
\frac{1}{\left|H^{F}\right|} \sum_{h \in H^{F}} f(h)=\sum_{\iota \in I(S)^{F}} \sum_{[u] \in \mathcal{U}\left(H_{\iota}^{F}\right)} \sum_{s \in S_{\iota}^{F}} \frac{1}{\left|\bar{N}_{H}(s, S)^{F}\right|} \frac{f(s u)}{\left|C_{H_{\iota}}(u)^{F}\right|} .
$$

5.3. Restriction of Deligne-Lusztig characters. We now consider the function $f$ arising in our multiplicity formula. Let $H, S$ be as above, let $T$ be an $F$-stable maximal torus of $G$, and let $\chi \in \operatorname{Irr}\left(T^{F}\right), \eta \in \operatorname{Irr}\left(S^{F}\right)$ be arbitrary characters.

Using the function $f: H^{F} \rightarrow \mathbb{C}$ given by

$$
f(h)=\overline{R_{T, \chi}^{G}(h)} \cdot R_{S, \eta}^{H}(h),
$$

we have

$$
\left\langle R_{T, \chi}^{G}, R_{S, \eta}^{H}\right\rangle_{H^{F}}=\frac{1}{\left|H^{F}\right|} \sum_{h \in H^{F}} f(h) .
$$

The map

$$
j_{G_{s}}: H^{1}\left(F, W_{G_{s}}\right) \longrightarrow H^{1}\left(F, W_{G}\right)
$$

defined in (2.2) depends only on $G_{s}$, so we set

$$
j_{G_{\iota}}:=j_{G_{s}}, \quad \text { for any } s \in S_{\iota} .
$$

We have an analogous map

$$
j_{H_{\iota}}: H^{1}\left(F, W_{H_{\iota}}\right) \longrightarrow H^{1}\left(F, W_{H}\right) .
$$

Likewise, the sets $\bar{N}_{G}(s, T)^{F}$ and $\bar{N}_{H}(s, S)^{F}$ depend only on $\iota$, so we now write

$$
\bar{N}_{G}(\iota, T)^{F}:=\bar{N}_{G}(s, T)^{F}, \quad \bar{N}_{H}(\iota, S)^{F}:=\bar{N}_{H}(s, S)^{F},
$$

for $s \in S_{\iota}^{F}$.

Using (4.5) for $G$ and $H$, along with (5.4), our multiplicity formula becomes

$$
\left\langle R_{T, \chi}^{G}, R_{S, \eta}^{H}\right\rangle_{H^{F}}=\sum_{\substack{\iota \in I(S)^{F} \\[u] \in \mathcal{U}\left(H_{\iota}^{F}\right)}} \sum_{v, \varsigma} \frac{\overline{Q_{T_{v}}^{G_{\iota}}(u)} Q_{S_{\varsigma}}^{H_{\iota}}(u)}{\left|\bar{N}_{H}(\iota, S)^{F}\right|\left|C_{H_{\iota}}(u)^{F}\right|} \sum_{s \in S_{\iota}^{F}} \overline{\chi_{v}(s)} \eta_{\varsigma}(s),
$$

where the middle sum runs over $v \in j_{G_{\iota}}^{-1}(\operatorname{cl}(T, G))$ and $\varsigma \in j_{H_{\iota}}^{-1}(\operatorname{cl}(S, H))$. The character sums $\chi_{v}$ and $\eta_{\varsigma}$ are as defined in (4.4). 
5.4. Green functions. We digress from our multiplicity formula (5.7) to recall the polynomial nature of Green functions $Q_{T}^{G}$, defined on the unipotent set of $G^{F}$, for a connected reductive f-group $G$ with Frobenius $F$ and $F$-stable maximal torus $T$ in $G$.

For $u=1$, we have

$$
Q_{T}^{G}(1)=\epsilon_{G}(w)\left[G^{F}: T^{F}\right]_{p^{\prime}},
$$

where $\left[G^{F}: T^{F}\right]_{p^{\prime}}$ is the maximal divisor of the index $\left[G^{F}: T^{F}\right]$ which is prime to $p, w \in \operatorname{cl}(T, G)$ and $\epsilon_{G}: W_{G} \rightarrow\{ \pm 1\}$ is the sign character of $W_{G}$. Note that $\epsilon_{G}(w)=(-1)^{\mathrm{rk} G+\mathrm{rk} T}[5,7.5 .2]$.

For $u \neq 1$, the Green functions $Q_{T}^{G}(u)$ can be expressed as polynomials which are known explicitly by tables for exceptional groups [3, [18] and for classical groups by recursive formulas [19] which can be implemented on a computer [9]. It will suffice for us to know the leading terms of these Green polynomials, which can be expressed in a uniform way.

Let $\mathcal{B}_{G}$ be the variety of Borel subgroups of $G$, and let $\mathcal{B}_{G}^{u}$ be the variety of $u$ fixed points in $\mathcal{B}_{G}$. The irreducible components of $\mathcal{B}_{G}^{u}$ all have the same dimension, and we set

$$
d_{G}(u):=\operatorname{dim} \mathcal{B}_{G}^{u}
$$

Steinberg proved that

$$
2 d_{G}(u)=\operatorname{dim} C_{G}(u)-\overline{\mathrm{rk}} G,
$$

where $\overline{\mathrm{rk}} G$ is the absolute rank of $G$.

Assume that $p$ is a good prime for $G$. For each unipotent class $[u] \in \mathcal{U}\left(G^{F}\right)$ and twisted conjugacy class $[w]=\varsigma \in H^{1}\left(F, W_{G}\right)$, there is a polynomial

$$
Q_{w, u}(t)=Q_{\varsigma, u}(t) \in \mathbb{Z}[t],
$$

of degree at most $d_{G}(u)$, such that

$$
Q_{T}^{G}(u)=Q_{w, u}(q)
$$

if $\operatorname{cl}(T, G)=[w]$ (see [20] and the references therein).

The coefficient of $t^{d_{G}(u)}$ in $Q_{w, u}(t)$ is

$$
\operatorname{tr}\left[w, H^{2 d_{G}(u)}\left(\mathcal{B}_{G}^{u}\right)\right]
$$

where $w$ acts on the $\ell$-adic cohomology of $\mathcal{B}_{G}^{u}$ via the Springer construction (see [21, [14, [16]).

If we take $u=1$, then $d_{G}(1)=N$ is the number of positive roots of $G$ and

$$
Q_{w, 1}^{G}(t)=\epsilon_{G}(w) t^{N}+\text { lower powers of } t,
$$

which is easily seen to be consistent with (5.8).

Suppose now that we replace $F$ by $F^{\nu}$ for some $\nu \geq 1$. The $G^{F^{\nu}}$-class of $T$ is then represented by

$$
(w \vartheta)^{\nu} \cdot \vartheta^{-\nu} \in W_{G}
$$

where $\vartheta$ is the automorphism of $W_{G}$ induced by $F$. Suppose $\nu \equiv 1 \bmod m$, where $m$ is a positive integer divisible by the exponent of the finite group $W_{G} \rtimes\langle\vartheta\rangle$. This implies that $F^{\nu}=F$ on $W_{G}$ and that $(w \vartheta)^{\nu} \cdot \vartheta^{-\nu}=w$ for all $w \in W_{G}$. It follows that $H^{1}\left(F, W_{G}\right)=H^{1}\left(F^{\nu}, W_{G}\right)$ and that the class $\mathrm{cl}(T, G)$ is the same with respect to $F$ or $F^{\nu}$. 
Likewise, the class of $u$ in $G^{F}$ or $G^{F^{\nu}}$ is determined by the $G$-conjugacy class $C \subset G$ containing $u$, together with a class in $H^{1}\left(F, A_{G}(C)\right)$ or $H^{1}\left(F^{\nu}, A_{G}(C)\right)$, where $A_{G}(C)$ is the component group of the centralizer of some $F$-fixed element in $C$. As in the preceding paragraph, we may take $m$ sufficiently divisible so that $F^{\nu}=F$ on $A_{G}(C)$ and that the class of $u$ in $G^{F}$ or $G^{F^{\nu}}$ corresponds to the same class in $H^{1}\left(F, A_{G}(C)\right)$. We may choose $m$ so that this holds for every $C$, since there are finitely many unipotent classes.

Let $Q_{T, \nu}^{G}$ be the Green function for $T$ on $G^{F^{\nu}}$. For $m$ sufficiently divisible as in the previous two paragraphs and $\nu \equiv 1 \bmod m$ we have

$$
Q_{T, \nu}^{G}(u)=Q_{w, u}\left(q^{\nu}\right) .
$$

(Note the difficulty with the exceptional class in $E_{8}$ is avoided since our conditions on $m$ imply that $\nu$ is odd; see [20, Remark 6.2].)

5.5. A progression of powers of Frobenius. The indices of and terms of the summations in (5.7) depend on $F$, and we wish to remove this dependence for infinitely many powers of $F$, in order to represent the sum in (5.7) as the value of a rational function.

There is a positive integer $m$ such that $F^{m}$ acts trivially on the finite set $I(S)$ and the divisibility conditions on $m$ from the previous section hold when $G$ is replaced by $G_{\iota}$ or $H_{\iota}$ for every $\iota \in I(S)$.

In particular, $m$ is divisible by the orders of the component groups $A_{\iota}(u)$ of the centralizers in $H_{\iota}$ of all unipotent elements $u \in H_{\iota}^{F}$ for every $\iota \in I(S)^{F}$, and $F^{m}$ is the identity automorphism on $A_{\iota}(u)$ for all such $\iota$ and $u$. This implies that for each $\iota \in I(S)^{F}$ and $[u] \in \mathcal{U}\left(H_{\iota}^{F}\right)$, there is a polynomial $P_{\iota, u}(t) \in \mathbb{Z}[t]$, of degree equal to $\operatorname{dim} C_{H_{\iota}}(u)$, such that

$$
\left|C_{H_{\iota}}(u)^{F^{\nu}}\right|=P_{\iota, u}\left(q^{\nu}\right)
$$

for all $\nu \equiv 1 \bmod m$. Moreover, each polynomial $P_{\iota, u}(t)$ is of the form $\left|A_{\iota}(u)\right|$ times a monic polynomial in $\mathbb{Z}[t]$.

The above conditions on $m$ also ensure that the indices in the outer two summations in (5.7), as well as the quantity $\left|\bar{N}_{H}(\iota, S)^{F}\right|$ are unchanged if $F$ is replaced by $F^{\nu}$ for $\nu \equiv 1 \bmod m$.

To handle the inner sum, we add more conditions: in the next section we will define certain subgroups $Z_{J}$ of $S$, indexed by subsets $J \subset I(S)^{F}$. We also insist that $m$ be divisible by $\left|Z_{J} / Z_{J}^{\circ}\right|$ and that $F^{m}$ act trivially on $Z_{J} / Z_{J}^{\circ}$ for each $J \subset I(S)^{F}$.

5.6. Character sums. In order to interpret the inner sum of (5.7) as a rational function, we shall replace each summand $S_{\iota}^{F}$ by the group $Z_{\iota}^{F}$, where

$$
Z_{\iota}:=Z\left(G_{\iota}\right) \cap S \text {. }
$$

It is easy to check that

$$
\begin{aligned}
& Z_{\iota} \subset Z\left(H_{\iota}\right), \\
& S_{\iota} \subset Z_{\iota} \subset S, \\
& G_{\iota}=C_{G}\left(Z_{\iota}\right)^{\circ} .
\end{aligned}
$$


Let $\chi_{v}$ and $\eta_{\varsigma}$ be the character sums appearing in (5.7). Our aim is to express the sum

$$
\frac{1}{\left|Z_{\iota}^{F}\right|} \sum_{s \in S_{\iota}^{F}} \overline{\chi_{v}(s)} \eta_{\varsigma}(s)
$$

as the value of a rational function.

Define a partial ordering on $I(S)$ by

$$
\iota^{\prime} \leq \iota \quad \Leftrightarrow \quad G_{\iota} \subseteq G_{\iota^{\prime}}
$$

Equivalently, we have

$$
\iota^{\prime} \leq \iota \quad \Leftrightarrow \quad Z_{\iota^{\prime}} \subseteq Z_{\iota}
$$

Let

$$
Y_{\iota}:=Z_{\iota}-S_{\iota}
$$

be the complement of $S_{\iota}$ in $Z_{\iota}$.

Lemma 5.1. For every $\iota \in I(S)$ we have

$$
Y_{\iota}=\bigcup_{\iota^{\prime}<\iota} Z_{\iota^{\prime}} .
$$

Proof. Let $s \in Y_{\iota}$. Then $s \in S_{\iota^{\prime}}$ for some $\iota^{\prime} \in I(S)$, with $\iota^{\prime} \neq \iota$, so $s \in Z_{\iota^{\prime}}$. Since $Y_{\iota} \subset Z_{\iota}$, we have

$$
G_{\iota}=C_{G}^{\circ}\left(Z_{\iota}\right) \subset C_{G}^{\circ}\left(Y_{\iota}\right) \subseteq G_{s}=G_{\iota^{\prime}},
$$

so $\iota^{\prime}<\iota$.

Conversely, let $s \in Z_{\iota^{\prime}}$, with $\iota^{\prime}<\iota$. Note that $s \in Z_{\iota}$. If $s \notin Y_{\iota}$, then $s \in S_{\iota}$. This implies that

$$
G_{\iota^{\prime}}=C_{G}\left(Z_{\iota^{\prime}}\right)^{\circ} \subset G_{s}=G_{\iota},
$$

contradicting $\iota^{\prime}<\iota$. This proves the lemma.

For a subset $J \subseteq I(S)$, let

$$
Z_{J}=\bigcap_{\iota^{\prime} \in J} Z_{\iota^{\prime}}
$$

There is a polynomial $f_{J} \in \mathbb{C}[t]$ of degree $\operatorname{dim} Z_{J}$ such that

$$
f_{J}\left(q^{\nu}\right)=\left|Z_{J}^{F^{\nu}}\right|, \quad \text { for all } \nu \equiv 1 \bmod m .
$$

For $\nu \geq 1$, let

$$
N_{\nu}^{T_{v}}: T_{v}^{F^{\nu}} \longrightarrow T_{v}^{F}, \quad N_{\nu}^{S_{\varsigma}}: S_{\varsigma}^{F^{\nu}} \longrightarrow S_{\varsigma}^{F}
$$

be the norm mappings. These are surjective. Set

$$
\chi_{v}^{(\nu)}:=\chi_{v} \circ N_{\nu}^{T_{v}}, \quad \eta_{\varsigma}^{(\nu)}:=\eta_{\varsigma} \circ N_{\nu}^{S_{\varsigma}} .
$$

Assume that $\iota \in J \subset I(S)^{F}$. Then $Z_{J}$ is $F$-stable and

$$
Z_{J}^{F} \subset Z_{\iota}^{F} \subset Z\left(G_{\iota}\right)^{F} \cap Z\left(H_{\iota}\right)^{F} .
$$

Both $\chi_{v}$ and $\eta_{\varsigma}$ are defined on the latter group (see (4.4)), so we may restrict them to $Z_{J}^{F}$. Our conditions on $m$ at the end of section 5.5 ensure that the restricted norm mapping

$$
N_{\nu}^{S_{\varsigma}}: Z_{J}^{F^{\nu}} \longrightarrow Z_{J}^{F}
$$

is also surjective. This implies, for all integers $\nu \equiv 1 \bmod m$, that

$$
\left\langle\chi_{v}^{(\nu)}, \eta_{\varsigma}^{(\nu)}\right\rangle_{Z_{J}^{F^{\nu}}}=\left\langle\chi_{v}, \eta_{\varsigma}\right\rangle_{Z_{J}^{F}}
$$


Hence for each $J$, we have

$$
\sum_{z \in Z_{J}^{F^{\nu}}} \overline{\chi_{v}^{(\nu)}(z)} \eta_{\varsigma}^{(\nu)}(z)=\left\langle\chi_{v}, \eta_{\varsigma}\right\rangle_{Z_{J}^{F}} \cdot f_{J}\left(q^{\nu}\right)
$$

Let $I(\iota, S):=\left\{\iota^{\prime} \in I(S): \iota^{\prime}<\iota\right\}$. It now follows from Möbius inversion that the rational function

$$
\Theta_{\iota, v, \varsigma}(t):=\left\langle\chi_{v}, \eta_{\varsigma}\right\rangle_{Z_{\iota}^{F}}+\sum_{J \subseteq I(\iota, S)^{F}}(-1)^{|J|}\left\langle\chi_{v}, \eta_{\varsigma}\right\rangle_{Z_{J}^{F}} \frac{f_{J}(t)}{f_{\iota}(t)}
$$

has the property that

$$
\Theta_{\iota, v, \varsigma}\left(q^{\nu}\right)=\frac{1}{\left|Z_{\iota}^{F^{\nu}}\right|} \sum_{s \in S_{\iota}^{F^{\nu}}} \overline{\chi_{v}^{(\nu)}(s)} \eta_{\varsigma}^{(\nu)}(s)
$$

for all $\nu \equiv 1 \bmod m$. Since $\operatorname{dim} Z_{J} \leq \operatorname{dim} Z_{\iota}$ for all $J \subseteq I(\iota, S)^{F}$, we have

$$
\operatorname{deg} \Theta_{\iota, v, \varsigma} \leq 0 \text {. }
$$

5.7. Multiplicity as a polynomial. We return to our multiplicity formula (5.7). We have shown that

$$
\left\langle R_{T, \chi}^{G}, R_{S, \eta}^{H}\right\rangle_{H^{F}}=\sum_{\alpha} \Psi_{\alpha}(q) \Theta_{\alpha}(q),
$$

where $\alpha$ runs over quadruples $\alpha=(\iota, u, v, \varsigma)$, with

$$
\iota \in I(S)^{F}, \quad[u] \in \mathcal{U}\left(H_{\iota}^{F}\right), \quad v \in j_{G_{\iota}}^{-1}(\operatorname{cl}(T, G)), \quad \varsigma \in j_{H_{\iota}}^{-1}(\operatorname{cl}(S, H)),
$$

$\Theta_{\alpha}(t)=\Theta_{\iota, v, \varsigma}(t)$ is the rational function defined in (5.16) and $\Psi_{\alpha}(t)$ is the rational function defined by

$$
\Psi_{\alpha}(t)=f_{\iota}(t) \cdot \frac{\overline{Q_{v, u}^{G_{\iota}}(t)} Q_{\varsigma, u}^{H_{\iota}}(t)}{\left|\bar{N}_{H}(\iota, S)^{F}\right|\left|P_{\iota, u}(t)\right|} .
$$

Here $Q_{v, u}^{G_{\iota}}(t)$ and $Q_{\varsigma, u}^{H_{\iota}}(t)$ are the Green polynomials from section 5.4 and $P_{\iota, u}(t)$ is the polynomial from (5.11).

If $F$ is replaced by $F^{\nu}$ with $\nu \equiv 1 \bmod m$, where $m$ is as in section 5.5 the summation indices $\alpha$ are unchanged, so that the rational function

$$
M(t):=\sum_{\alpha} \Psi_{\alpha}(t) \Theta_{\alpha}(t)
$$

has the property that

$$
\left\langle R_{T, \chi^{(\nu)}}^{G}, R_{S, \eta^{(\nu)}}^{H}\right\rangle_{H^{F^{\nu}}}=M\left(q^{\nu}\right),
$$

for all $\nu \equiv 1 \bmod m$. In particular, $M\left(q^{\nu}\right)$ is an integer for all $\nu \equiv 1 \bmod m$.

We next observe that the numerator of each term in $M(t)$ belongs to $\mathbb{Z}[t]$, and the denominator of each term in $M(t)$ is an integer times a monic polynomial in $\mathbb{Z}[t]$. Hence there is $a \in \mathbb{Z}$ such that

$$
a M(t)=\frac{f(t)}{g(t)},
$$

where $f(t)$ and $g(t)$ are in $\mathbb{Z}[t]$ and $g(t)$ is monic. We can therefore write

$$
a M(t)=p(t)+r(t)
$$


where $p(t) \in \mathbb{Z}[t]$ and $r(t)$ is a rational function of negative degree. On the other hand,

$$
r\left(q^{\nu}\right)=a M\left(q^{\nu}\right)-p\left(q^{\nu}\right)=a\left\langle R_{T, \chi^{(\nu)}}^{G}, R_{S, \eta^{(\nu)}}^{H}\right\rangle_{H^{F^{\nu}}}-p\left(q^{\nu}\right) \in \mathbb{Z}
$$

for all $\nu \equiv 1 \bmod m$. Since $r\left(q^{\nu}\right) \rightarrow 0$ as $\nu \rightarrow \infty$, we must have $r(t) \equiv 0$, so

$$
M(t)=\frac{1}{a} p(t) .
$$

This shows that $M(t)$ is a polynomial, as claimed.

\section{Complexity And the Degree of $M(t)$}

From now on, the algebraic group $G$ is simple. That is, the center $Z(G)$ is finite and contains every normal subgroup of $G$. Recall that the complexity $\delta$ is the minimum codimension of a $B_{H}$-orbit in $G / B$. In this section we will complete the proof of the first assertion of Theorem 1.4 by showing that $\delta$ is an upper bound on the degree of the multiplicity polynomial $M(t)$ defined in (5.21).

6.1. A formula for the complexity. In this section we show that $\delta$ has the simplest conceivable formula. Let $\mathfrak{g}$ and $\mathfrak{h}$ be the Lie algebras of $G$ and $H$. We are assuming that $\mathfrak{g}$ is simple. We also invoke Assumption 1.3. That is, we assume that $\mathfrak{g}=\mathfrak{h} \oplus \mathfrak{m}$, stable under $\operatorname{Ad}(H)$, and that there is a nondegenerate $\operatorname{Ad}(H)$-invariant symmetric form $B$ on $\mathfrak{m}$. Hence Ad restricts to a homomorphism

$$
\text { Ad }: H \longrightarrow S O(\mathfrak{m}) \text {. }
$$

Lemma 6.1. Assume that $H \neq G$. Then

$$
\operatorname{ker}[\text { Ad }: H \rightarrow S O(\mathfrak{m})]=Z(G) \cap H .
$$

Proof. Containment " $\supseteq$ " is clear. We prove containment " $\subseteq$ ". Set

$$
N:=\operatorname{ker}[\operatorname{Ad}: H \rightarrow S O(\mathfrak{m})]
$$

and let $\mathfrak{n}$ be the Lie algebra of $N$. We have

$$
\mathfrak{n}=\operatorname{ker}[\operatorname{ad}: \mathfrak{h} \rightarrow \mathfrak{s o}(\mathfrak{m})],
$$

so $\mathfrak{n}$ is an ideal in $\mathfrak{h}$. But $[\mathfrak{n}, \mathfrak{m}]=0$, so $\mathfrak{n}$ is in fact an ideal in $\mathfrak{g}$. Since $\mathfrak{g}$ is simple and not equal to $\mathfrak{h}$, we have $\mathfrak{n}=0$. Hence $N$ is a finite normal algebraic subgroup of $H$. By [4, 22.1], $N$ is central in $H$, hence $\operatorname{Ad}(N)$ acts trivially on $\mathfrak{h}$, as well as on $\mathfrak{m}$. It follows that $N$ is central in $G$. This completes the proof.

Let $B$ and $B_{H}$ be Borel subgroups of $G$ and $H$, respectively. Let $U$ and $V$ be their respective unipotent radicals. After conjugating, we may assume that

$$
B_{H}=S V, \quad B=T U
$$

with

$$
S \subset T, \quad V \subset U .
$$

Proposition 6.2. The complexity $\delta$ is given by

$$
\delta= \begin{cases}\operatorname{dim} G / B-\operatorname{dim} B_{H} & \text { if } H \neq G, \\ 0 & \text { if } H=G .\end{cases}
$$


Proof. If $H=G$, the fact that $\delta=0$ is clear from the Bruhat decomposition. Assume from now on that $H \neq G$. We must show that $B_{H}$ has an orbit in $G / B$ with finite stabilizers. Let $w$ be the element of $W_{G}(T)$ such that ${ }^{w} B \cap B=T$. Then every element of $U w B / B$ can be uniquely expressed as $u w B$ for $u \in U$. For $v \in V, s \in S$, we have

$$
v s \cdot u w B=v\left(\text { sus }^{-1}\right) w B .
$$

By uniqueness of expression, $v s$ fixes $u w B$ if and only if $v=u s u^{-1} s^{-1}$. It follows that the projection $B_{H} \rightarrow S$ gives an isomorphism from the $B_{H}$-stabilizer of $u w B$ to the $S$-stabilizer of $u^{-1} V$ in the quotient variety $U / V$. We will show there exists $u \in U$ such that the latter stabilizer is finite.

Denote the Lie algebras of $U, V, T, S$ by $\mathfrak{u}, \mathfrak{v}, \mathfrak{t}, \mathfrak{s}$. The tangent space to $U / V$ at $\mathrm{eV}$ is $\mathfrak{u} / \mathfrak{v}$. We have

$$
\mathfrak{g} / \mathfrak{h}=\mathfrak{t} / \mathfrak{s} \oplus \mathfrak{u} / \mathfrak{v} \oplus \overline{\mathfrak{u}} / \overline{\mathfrak{v}},
$$

where $\overline{\mathfrak{u}}=\operatorname{Ad}(w) \mathfrak{u}$ is the opposite nilradical of $\mathfrak{u}$ and $\overline{\mathfrak{v}}$ is the opposite nilradical of $\mathfrak{v}$.

Since $\operatorname{ker}[\operatorname{Ad}: S \rightarrow G L(\mathfrak{g} / \mathfrak{h})]$ is finite by Lemma 6.1, it follows that $\operatorname{ker}[\operatorname{Ad}:$ $S \rightarrow G L(\mathfrak{u} / \mathfrak{v})]$ is finite. This latter kernel is the set of common zeros of the roots $\Phi(S, U / V)$ of $S$ in $\mathfrak{u} / \mathfrak{v}$ (see [4, 8.17]). We have

$$
\mathfrak{u} / \mathfrak{v}=(\mathfrak{u} / \mathfrak{v})^{S} \oplus \sum_{\alpha \in \Phi(S, U / V)}(\mathfrak{u} / \mathfrak{v})_{\alpha} .
$$

A vector in $\mathfrak{u} / \mathfrak{v}$ whose $\alpha$-component is nonzero for every $\alpha \in \Phi(S, U / V)$ will therefore have finite stabilizer in $S$. Proposition 6.2 now follows from a basic result:

Lemma 6.3. Let $k$ be an algebraically closed field. Suppose a $k$-torus $S$ acts on a smooth irreducible affine $k$-variety $X$, fixing a point $x \in X$, so that $S$ acts on the tangent space $T_{x} X$ at $x$. If there exists $v \in T_{x} X$ having finite stabilizer $S_{v} \subset S$, then there exists $y \in X$ having finite stabilizer $S_{y} \subset S$.

This lemma can be proved as follows. Since the torus $S$ acts completely reducibly on the coordinate ring $k[X]$, the argument of Lemme 1 in [15] shows that there is an $S$-equivariant morphism $\varphi: X \rightarrow T_{x} X$ such that $\varphi(x)=0$, and whose differential $d \varphi_{x}: T_{x} X \rightarrow T_{x} X$ is bijective. The set $\mathcal{U}$ of points in $T_{x} X$ with finite stabilizers is open, and nonempty by hypothesis. Since $\varphi$ is dominant, the preimage $\varphi^{-1}(\mathcal{U})$ is nonempty. If $y \in \varphi^{-1}(\mathcal{U})$, then $S_{y} \subseteq S_{\varphi(y)}$, and the latter stabilizer is finite.

Lemma 6.3 can also be proved using a $T$-equivariant embedding of $X$ in a linear representation of $T$.

6.2. Degree of $\Psi_{\alpha}(t)$. We return now to our rational function

$$
\Psi_{\alpha}(t)=f_{\iota}(t) \cdot \frac{\overline{Q_{v, u}^{G_{\iota}}(t)} Q_{\varsigma, u}^{H_{\iota}}(t)}{\left|\bar{N}_{H}(\iota, S)^{F}\right|\left|P_{\iota, u}(t)\right|} .
$$

We have

$$
\operatorname{deg}\left|P_{\iota, u}(t)\right|=\operatorname{dim} C_{H_{\iota}}(u), \quad \operatorname{deg}\left|f_{\iota}(t)\right|=\operatorname{dim} Z_{\iota} .
$$

From section 5.4 and equation (5.9) we find that

$$
\begin{aligned}
\operatorname{deg} \Psi_{\alpha}(t) & \leq \operatorname{dim} Z_{\iota}+d_{G_{\iota}}(u)+d_{H_{\iota}}(u)-\operatorname{dim} C_{H_{\iota}}(u) \\
& =\operatorname{dim} Z_{\iota}+\frac{1}{2}\left[\operatorname{dim} C_{G_{\iota}}(u)-\operatorname{dim} C_{H_{\iota}}(u)-\overline{\mathrm{rk}} G-\overline{\mathrm{rk}} H\right] .
\end{aligned}
$$


The fixed point spaces $\mathfrak{g}^{s}, \mathfrak{h}^{s}, \mathfrak{m}^{s}$ are the same for any $s \in S_{\iota}$; we denote them by $\mathfrak{g}_{\iota}, \mathfrak{h}_{\iota}, \mathfrak{m}_{\iota}$. Thus we have an $\operatorname{Ad}(H)$-stable decomposition

$$
\mathfrak{g}_{\iota}=\mathfrak{h}_{\iota} \oplus \mathfrak{m}_{\iota}
$$

and

$$
\begin{aligned}
\operatorname{dim} C_{G_{\iota}}(u)-\operatorname{dim} C_{H_{\iota}}(u) & =\operatorname{dim} \mathfrak{m}_{\iota}^{u} \\
& \leq \operatorname{dim} \mathfrak{m}_{\iota} \\
& =\operatorname{dim} C_{G_{\iota}}(1)-\operatorname{dim} C_{H_{\iota}}(1) .
\end{aligned}
$$

Define

$$
\begin{aligned}
\delta_{\iota} & :=\operatorname{dim} Z_{\iota}+\operatorname{dim} \mathcal{B}_{G_{\iota}}-\operatorname{dim} \mathcal{B}_{H_{\iota}}-\operatorname{dim} S \\
& =\operatorname{dim} Z_{\iota}+\frac{1}{2}\left[\operatorname{dim} \mathfrak{m}_{\iota}-\overline{\mathrm{rk}} G-\overline{\mathrm{rk}} H\right] .
\end{aligned}
$$

For example, if $\iota_{0}$ is the minimal element of $I(S)$, then

$$
G_{\iota_{0}}=G, \quad Z_{\iota_{0}}=Z(G) \cap S \quad \text { and } \mathfrak{m}_{\iota_{0}}=\mathfrak{m} .
$$

Since $Z(G)$ is finite, Proposition 6.2 implies that

$$
\delta_{\iota_{0}}=\frac{1}{2}[\operatorname{dim} \mathfrak{m}-\overline{\mathrm{rk}} G-\overline{\mathrm{rk}} H]=\delta, \quad \text { if } H \neq G .
$$

Lemma 6.4. We have $\operatorname{deg} \Psi_{\alpha}(t) \leq \delta_{\iota}$, with equality only if $u=1$.

Proof. The inequality follows from (6.2) and (6.3), and the last assertion follows from section 6.1.

We now seek a bound on $\operatorname{deg} \Psi_{\alpha}$ which is independent of $\iota$. We will show that $\delta_{\iota} \leq \delta$, and that equality holds only in rather special circumstances.

Let $\mathfrak{m}_{\iota}^{\prime}$ be the sum of the eigenspaces of $\operatorname{Ad}(s)$ in $\mathfrak{m}$ with eigenvalues $\neq 1$, for any $s \in S_{\iota}$. Since $\operatorname{det} \operatorname{Ad}(H)=1$ on $\mathfrak{m}$, the dimension $\operatorname{dim} \mathfrak{m}_{\iota}^{\prime}$ is even. We have $\mathfrak{m}=\mathfrak{m}_{\iota} \oplus \mathfrak{m}_{\iota}^{\prime}$, the form $B$ is nondegenerate on $\mathfrak{m}_{\iota}^{\prime}$, and Ad $: H \rightarrow S O(\mathfrak{m})$ restricts to a homomorphism $\mathrm{Ad}_{\iota}: H_{\iota} \rightarrow S O\left(\mathfrak{m}_{\iota}^{\prime}\right)$.

Lemma 6.5. For every $\iota \in I(S)$ we have $\delta_{\iota} \leq \delta$. Moreover, if $H \neq G$, then the following are equivalent:

(1) $\delta_{\iota}=\delta$;

(2) $\operatorname{dim}\left(Z_{\iota}\right)=\frac{1}{2} \operatorname{dim} \mathfrak{m}_{\iota}^{\prime}$;

(3) $\operatorname{Ad}_{\iota}\left(Z_{\iota}\right)$ is a maximal torus in $S O\left(\mathfrak{m}_{\iota}^{\prime}\right)$.

When these hold, the derived group of $H_{\iota}$ acts trivially on $\mathfrak{m}_{\iota}^{\prime}$.

Proof. If $H=G$, then $\delta=0$ and

$$
\delta_{\iota}=\operatorname{dim} Z_{\iota}-\operatorname{dim} S \leq 0 .
$$

From now on assume $H \neq G$. From (6.4) and (6.6), we have

$$
\delta-\delta_{\iota}=\frac{1}{2} \operatorname{dim} \mathfrak{m}_{\iota}^{\prime}-\operatorname{dim} Z_{\iota} \text {. }
$$

Now, the group

$$
N_{\iota}:=\operatorname{ker}\left[\operatorname{Ad}_{\iota}: Z_{\iota} \longrightarrow S O\left(\mathfrak{m}_{\iota}^{\prime}\right)\right]
$$

is finite. Indeed, since $Z_{\iota} \subset Z\left(G_{\iota}\right)$, it follows that $N_{\iota}$ centralizes $\mathfrak{m}_{\iota}$, as well as $\mathfrak{m}_{\iota}^{\prime}$. Hence we have

$$
N_{\iota} \subseteq \operatorname{ker}[\text { Ad }: H \longrightarrow S O(\mathfrak{m})]=Z(G) \cap H,
$$

the latter equality being from Lemma 6.1. Hence $N_{\iota} \subset Z(G)$, and the latter is finite since $G$ is simple. 
Since $\operatorname{Ad}\left(Z_{\iota}^{\circ}\right)$ is a torus in $S O\left(\mathfrak{m}_{\iota}^{\prime}\right)$ and $\frac{1}{2} \operatorname{dim} \mathfrak{m}_{\iota}^{\prime}$ is the dimension of a maximal torus in $S O\left(\mathfrak{m}_{\iota}^{\prime}\right)$, this proves that both sides of (6.7) are $\geq 0$ and that (1)-(3) are equivalent.

For the last assertion, recall that $Z_{\iota} \subset Z\left(H_{\iota}\right)$. If (1)-(3) hold, then $\operatorname{Ad}_{\iota}\left(H_{\iota}\right)$ centralizes a maximal torus in $S O\left(\mathfrak{m}_{\iota}^{\prime}\right)$, hence is contained in that torus.

With this lemma, the first assertion of Theorem 1.4 has been proved.

6.3. A remark on the multiplicity formula. The formula (5.21), as written, contains more terms than are necessary. For, if we write

$$
\Psi_{\alpha}(t) \Theta_{\alpha}(t)=P_{\alpha}(t)+R_{\alpha}(t),
$$

where $P_{\alpha}(t)$ is a polynomial and $\operatorname{deg} R_{\alpha}(t)<0$, then

$$
M(t)=\sum_{\alpha} P_{\alpha}(t) \quad \text { and } \quad \sum_{\alpha} R_{\alpha}(t)=0,
$$

since $M(t)$ is a polynomial. From (5.17) and Lemma 6.4 we have $\operatorname{deg} P_{\alpha} \leq \delta_{\iota}$, where $\alpha=(\iota, u, v, \varsigma)$. It follows that

$$
\left\langle R_{T, \chi}^{G}, R_{S, \eta}^{H}\right\rangle_{H^{F}}=M(q)=\sum_{\alpha} P_{\alpha}(q),
$$

where the sum is over just those $\alpha=(\iota, u, v, \varsigma)$ such that $\delta_{\iota} \geq 0$.

\section{The Leading term of $M(t)$}

We have shown that the multiplicity polynomial $M(t)$ has the form

$$
M(t)=A t^{\delta}+(\text { lower powers of } t) .
$$

In this section we find an explicit and effective formula for the leading term $A$ of $M(t)$. Recall from (5.21) that

$$
M(t)=\sum_{\alpha} \Psi_{\alpha}(t) \Theta_{\alpha}(t),
$$

where $\alpha$ runs over quadruples $(\iota, u, v, \varsigma)$ as in (5.19),

$$
\Psi_{\alpha}(t)=f_{\iota}(t) \cdot \frac{\overline{Q_{v, u}^{G_{\iota}}(t)} Q_{\varsigma, u}^{H_{\iota}}(t)}{\left|\bar{N}_{H}(\iota, S)^{F}\right|\left|P_{\iota, u}(t)\right|}
$$

and

$$
\Theta_{\alpha}(t)=\left\langle\chi_{v}, \eta_{\varsigma}\right\rangle_{Z_{\iota}^{F}}+\sum_{J \subseteq I(\iota, S)^{F}}(-1)^{|J|}\left\langle\chi_{v}, \eta_{\varsigma}\right\rangle_{Z_{J}^{F}} \frac{f_{J}(t)}{f_{\iota}(t)} .
$$

By Lemmas 6.4 and 6.5. only quadruples $\alpha$ with $u=1$ and $\delta_{\iota}=\delta$ contribute to the leading term; henceforth we assume $\alpha$ is of this form. As a power series in $t$, we then have

$$
\Psi_{\alpha}(t)=A_{\alpha} t^{\delta}+(\text { lower degree terms }),
$$

where

$$
A_{\alpha}=\left[Z_{\iota}^{F}: Z_{\iota}^{\circ F}\right] \cdot \frac{(-1)^{\mathrm{rk}\left(G_{\iota}\right)+\mathrm{rk}(T)+\mathrm{rk}\left(H_{\iota}\right)+\mathrm{rk}(S)}}{\left|\bar{N}_{H}(\iota, S)^{F}\right|} .
$$

At first glance, each function $\Theta_{\alpha}(t)$ could contribute many terms to $A$, coming from various $\iota^{\prime}<\iota$ with $\operatorname{dim} Z_{\iota^{\prime}}=\operatorname{dim} Z_{\iota}$, since $Z_{\iota}$ may be disconnected. We now show that in fact $\Theta_{\alpha}(t)$ contributes only one term. 
Lemma 7.1. If $\delta_{\iota}=\delta$ and $\iota^{\prime}<\iota$, then $\operatorname{dim} Z_{\iota^{\prime}}<\operatorname{dim} Z_{\iota}$.

Proof. If $H=G$, we have $\delta_{\iota}=\operatorname{dim} Z_{\iota}-\operatorname{dim} S \leq 0=\delta$ with equality iff $Z_{\iota}=S$. The lemma holds since $S$ is connected.

Now assume $H \neq G$. Suppose $\delta_{\iota}=\delta$ and $\iota^{\prime}<\iota$, yet $\operatorname{dim} Z_{\iota^{\prime}}=\operatorname{dim} Z_{\iota}$. Then

$$
Z_{\iota}^{\circ} \subseteq Z_{\iota^{\prime}} \subset Z_{\iota} \text {. }
$$

From Lemma 6.5, the image $\operatorname{Ad}_{\iota}\left(Z_{\iota}\right)$ is a maximal torus in $S O\left(\mathfrak{m}_{\iota}^{\prime}\right)$. It follows that

$$
\operatorname{Ad}_{\iota}\left(Z_{\iota}^{\circ}\right)=\operatorname{Ad}\left(Z_{\iota}\right) .
$$

Thus, for each $z \in Z_{\iota}$ there is $z_{0} \in Z_{\iota}^{\circ}$ such that

$$
z_{1}:=z z_{0}^{-1} \in \operatorname{ker}\left[\operatorname{Ad}_{\iota}: Z_{\iota} \rightarrow S O\left(\mathfrak{m}_{\iota}^{\prime}\right)\right] .
$$

By Lemma 6.1, we have $z_{1} \in Z(G) \cap H$. Hence

$$
z=z_{0} z_{1} \in Z_{\iota}^{\circ} \cdot(Z(G) \cap H) .
$$

We have shown that

$$
Z_{\iota}=Z_{\iota}^{\circ} \cdot(Z(G) \cap H) .
$$

Now $S_{\iota}$ is stable under multiplication by $Z(G) \cap H$. Moreover, $S_{\iota}$ is open in $Z_{\iota}$, so $S_{\iota}$ meets some connected component of $Z_{\iota}$ in an open dense set. But then (7.4) implies that $S_{\iota}$ meets every connected component of $Z_{\iota}$ in an open dense set.

Likewise, $S_{\iota^{\prime}}$ meets some component of $Z_{\iota^{\prime}}$ in an open dense set. By (7.2), every such component of $Z_{\iota^{\prime}}$ is also a component of $Z_{\iota}$. Therefore $S_{\iota}$ and $S_{\iota^{\prime}}$ meet a common component of $Z_{\iota}$ in a dense open set. This implies that $S_{\iota} \cap S_{\iota^{\prime}}$ is nonempty; hence $\iota=\iota^{\prime}$, contradicting $\iota^{\prime}<\iota$.

As an aside, we mention the following consequence of (7.4) which simplifies our eventual formula for $A$ when $G$ is adjoint.

Lemma 7.2. Suppose $G$ is simple adjoint. If $\delta_{\iota}=\delta$, then $Z_{\iota}$ is connected.

Return now to $\Theta_{\alpha}(t)$. For each $J \subseteq I(\iota, S)$, the subgroup $Z_{J}$ is contained in some $Z_{\iota^{\prime}}$ with $\iota^{\prime}<\iota$. Lemma 7.1 implies that

$$
\operatorname{deg} f_{J}(t)<\operatorname{deg} f_{\iota}(t)
$$

which shows that the leading term of $\Theta_{\alpha}(t)$ has the following simple form.

Corollary 7.3. Let $\alpha=(\iota, 1, v, \varsigma)$ be a quadruple appearing in $M(t)$ with $\delta_{\iota}=\delta$. Then

$$
\Theta_{\alpha}(\infty)=\left\langle\chi_{v}, \eta_{\varsigma}\right\rangle_{Z_{\iota}^{F}}
$$

From (7.1) and Corollary 7.3 we get the following expression for the leading term A.

Proposition 7.4. The leading term $A$ of $M(t)$ in Theorem 1.4 is given by $A=$ $\sum_{\iota} A_{\iota}$, where $\iota$ runs over those $\iota \in I(S)^{F}$ with $\delta_{\iota}=\delta$, and

$$
A_{\iota}=(-1)^{\mathrm{rk}\left(G_{\iota}\right)+\mathrm{rk}(T)+\mathrm{rk}\left(H_{\iota}\right)+\mathrm{rk}(S)} \cdot \frac{\left|Z_{\iota}^{F} / Z_{\iota}^{\circ F}\right|}{\left|\bar{N}_{H}(\iota, S)\right|} \cdot \sum_{v, \varsigma}\left\langle\chi_{v}, \eta_{\varsigma}\right\rangle_{Z_{\iota}^{F}} .
$$

In the last summation, $v$ and $\varsigma$ run over $j_{G_{\iota}}^{-1}(\operatorname{cl}(T, G))$ and $j_{H_{\iota}}^{-1}(\operatorname{cl}(S, H))$, respectively. 
As a simple illustration of Proposition 7.4 we show how it reduces to the DeligneLusztig inner-product formula [8, thm. 6.8] when $G=H$. For $\iota \in I(S)$, we have then

$$
\delta_{\iota}=\operatorname{dim} Z\left(G_{\iota}\right)-\operatorname{dim} S \leq 0=\delta
$$

with equality iff $G_{\iota}=S=H_{\iota}$. This means $\iota$ is the maximal element of $I(S)^{F}$, and $M(t)=A=A_{\iota}$ is the inner product $\left\langle R_{T, \chi}^{G}, R_{S, \eta}^{G}\right\rangle_{G^{F}}$.

By Proposition 7.4, if $T$ is not $G^{F}$ conjugate to $S$, then $j_{G_{L}}^{-1}(\operatorname{cl}(T, G))=\varnothing$, so $A=0$. Otherwise we may take $S=T$, and the fiber of $j_{G_{\iota}}$ over $\operatorname{cl}(S, G)$ is the singleton $\{v\}$ corresponding to the class of $S$ in itself. We have

$$
\chi_{v}=\sum_{w \in W_{G}(S)^{F}}{ }^{w} \chi, \quad \eta_{v}=\sum_{w \in W_{G}(S)^{F}}{ }^{w} \eta, \quad \bar{N}_{G}(\iota, S)^{F}=W_{G}(S)^{F},
$$

and the result:

$$
A=\frac{\left\langle\chi_{v}, \eta_{v}\right\rangle_{S^{F}}}{\left|W_{G}(S)^{F}\right|}
$$

is the original Deligne-Lusztig formula for $\left\langle R_{S, \chi}^{G}, R_{S, \eta}^{G}\right\rangle_{G^{F}}$.

\section{Optimality}

Recall that $G$ is simple. In this section we show that the degree $\delta$ is optimal. We may assume that $H \neq G$. Let $T \subset G$ and $S \subset H$ be arbitrary $F$-stable maximal tori. We will show that for sufficiently large $q$, there are characters $\chi \in \operatorname{Irr}\left(T^{F}\right)$ and $\eta \in \operatorname{Irr}\left(S^{F}\right)$ such that the leading coefficient $A$ is nonzero. In fact, we can take $\eta$ to be the trivial character.

For each $\iota \in I(S)^{F}$ with $A_{\iota} \neq 0$, the fiber $j_{G_{\iota}}^{-1}(\operatorname{cl}(T, G))$ is nonempty. This means that $Z_{\iota}$ is $G^{F}$-conjugate to a subgroup $\tilde{Z}_{\iota} \subset T$. There are only finitely many of these subgroups $\tilde{Z}_{\iota}$. Recall from (6.5) that $\iota_{0} \in I(S)^{F}$ is the minimal element, for which $Z_{\iota 0}=Z(G) \cap S$. If $\operatorname{dim} Z_{\iota}=0$ and $\delta_{\iota}=\delta$, then Lemma 7.1 implies that $\iota=\iota_{0}$. Hence, if $\iota \neq \iota_{0}$, the torus $T / \tilde{Z}_{\iota}^{\circ}$ has strictly smaller dimension than that of $T$, so that $\left|\operatorname{Irr}\left(T^{F} / \tilde{Z}_{\iota}^{\circ F}\right)\right|$ is a polynomial in $q$ of degree strictly less than $\operatorname{dim} T$. Hence for sufficiently large $q$ there are characters $\chi \in \operatorname{Irr}\left(T^{F}\right)$ which are trivial on $Z_{\iota_{0}}^{F}$ and nontrivial on every $\tilde{Z}_{\iota}^{F}$ for $\iota \neq \iota_{0}$. We call these $\chi$ very regular. For very regular $\chi$ and $\iota$ such that $A_{\iota} \neq 0$, we have

$$
\left\langle\chi_{v}, 1\right\rangle_{Z_{\iota}^{F}}= \begin{cases}1 & \text { if } \iota=\iota_{0} \\ 0 & \text { if } \iota \neq \iota_{0} .\end{cases}
$$

It follows that for $\chi$ very regular, and $\eta=1$, the coefficient $A$ of $t^{\delta}$ in $M(t)$ is given by

$$
A=\epsilon_{G}(x) \epsilon_{H}(y),
$$

where $x \in \operatorname{cl}(T, G)$ and $y \in \operatorname{cl}(S, H)$.

Let $\vartheta$ be the automorphism of $W_{H}$ induced by $F$ and let $\psi$ be the character of an irreducible representation of $\langle\vartheta\rangle \ltimes W_{H}$. For each $y \in W_{H}$, choose an $F$-stable torus $S_{y}$ in $H$ such that $y \in \operatorname{cl}\left(S_{y}, H\right)$. We have then a class function $R_{\psi}^{H}$ of $H^{F}$ defined by

$$
R_{\psi}^{H}=\frac{1}{\left|W_{H}\right|} \sum_{y \in W_{H}} \psi(\vartheta y) R_{S_{y}, 1}^{H}
$$


For example, the trivial (1) and sign $\left(\epsilon_{H}\right)$ characters of $W_{H}$ extend to $\langle\vartheta\rangle \ltimes W_{H}$ (trivially on $\vartheta$ ). It is known (cf. [5, 7.6]) that $R_{1}^{H}=1_{H}$ and $R_{\epsilon_{H}}^{H}=S t_{H}$ are the trivial and Steinberg characters of $H^{F}$, respectively. For very regular $\chi$, (8.2) implies that

$$
\left\langle R_{T, \chi}^{G}, R_{\psi}^{H}\right\rangle_{H^{F}}=\epsilon_{G}(x)\left\langle\epsilon_{H}, \psi\right\rangle_{W_{H}} \cdot q^{\delta}+\text { (lower powers of } q \text { ). }
$$

In particular, we have

$$
\left\langle\epsilon_{G}(x) R_{T, \chi}^{G}, S t_{H}\right\rangle_{H^{F}}=q^{\delta}+(\text { lower powers of } q),
$$

while $\left\langle\epsilon_{G}(x) R_{T, \chi}^{G}, 1_{H}\right\rangle_{H^{F}}$ has degree $<\delta$. This last result is to be expected, in view of the results in [2] and [17.

\section{Restriction From $S_{2 n+1}$ to $S_{2 n}$.}

We return to the situation at the beginning of the introduction. So $p>2$ and $(V, Q)$ is a $(2 n+1)$-dimensional quadratic $\mathfrak{F}$-space, defined over $\mathfrak{f}$, with Frobenius $F$. Fix $v \in V^{F}$ with $Q(v) \neq 0$, and let $U$ be the orthogonal space of $v$ in $V$. We take

$$
G=S O(V), \quad H=G_{v}=S O(U),
$$

with $\mathfrak{f}$-structure on both groups induced from that on $V$. Assumption 1.3 holds: we may identify the quadratic spaces $(\mathfrak{m}, B)=(U, Q)$.

Let $T, S$ be $F$-stable maximal tori in $G$ and $H$ respectively, and let $\chi \in \operatorname{Irr}\left(T^{F}\right)$, $\eta \in \operatorname{Irr}\left(S^{F}\right)$ be characters, which for the moment are arbitrary.

We have

$$
\delta=\operatorname{dim} \mathcal{B}_{G}-\operatorname{dim} \mathcal{B}_{H}-\operatorname{dim} \overline{\mathrm{rk}} H=n^{2}-\left(n^{2}-n\right)-n=0 .
$$

From now on, we only consider $\iota \in I(S)^{F}$ with $\delta_{\iota}=0$. Since $G$ is adjoint, each such $Z_{\iota}$ is connected, by Lemma 7.2 Proposition 7.4 gives the multiplicity formula

$$
(-1)^{\mathrm{rk} T+\mathrm{rk} S}\left\langle R_{T, \chi}^{G}, R_{S, \eta}^{H}\right\rangle_{H^{F}}=\sum_{\substack{\iota \in I(S)^{F} \\ \delta_{\iota}=0}} \frac{(-1)^{\mathrm{rk}\left(G_{\iota}\right)+\mathrm{rk}\left(H_{\iota}\right)}}{\left|\bar{N}_{H}(\iota, S)\right|} \cdot \sum_{v, \varsigma}\left\langle\chi_{v}, \eta_{\varsigma}\right\rangle_{Z_{\iota}^{F}},
$$

where $v$ and $\varsigma$ run over $j_{G}^{-1}(\operatorname{cl}(T, G))$ and $j_{H_{\iota}}^{-1}(\operatorname{cl}(S, H))$, respectively.

The connectedness of $Z_{\iota}$ implies that -1 is not an eigenvalue of any $s \in S_{\iota}$. The last assertion of Lemma 6.5 implies that $s \in S_{\iota}$ has distinct eigenvalues on $V / V^{s}$. It follows that

$$
G_{\iota}=S O\left(V^{s}\right) \times Z_{\iota}, \quad H_{\iota}=S O\left(U^{s}\right) \times Z_{\iota} .
$$

Note that $\operatorname{dim} V^{s}$ is odd, say $\operatorname{dim} V^{s}=2 a+1$.

The decompositions (9.2) imply that if two $F$-stable maximal tori in $G_{\llcorner}^{F}$ are $G^{F}$-conjugate, then they are $G_{\iota}^{F}$-conjugate, and likewise for $H$. In other words, we have

$$
\left|j_{G_{\iota}}^{-1}(\operatorname{cl}(T, G))\right| \cdot\left|j_{H_{\iota}}^{-1}(\operatorname{cl}(S, H))\right| \leq 1 .
$$

Hence the inner sum of (9.1) has at most one term.

To make this precise, we recall that tori in orthogonal groups are described by pairs $\left(\lambda, \lambda^{\prime}\right)$ of partitions. We write partitions as $\lambda=\left(j^{\lambda_{j}}\right)$, meaning that 
$\lambda$ has $\lambda_{j}$ parts equal to $j$, and set $|\lambda|=\sum_{j} j \lambda_{j}$. We have pairs of partitions $\left(\nu, \nu^{\prime}\right),\left(\lambda, \lambda^{\prime}\right),\left(\mu, \mu^{\prime}\right)$ such that

$$
\begin{array}{rlrl}
Z_{\iota}^{F} & \simeq \prod_{j}\left(\mathfrak{f}_{j}^{\times}\right)^{\nu_{j}} \times\left(\mathfrak{f}_{2 j}^{1}\right)^{\nu_{j}^{\prime}}, & & |\nu|+\left|\nu^{\prime}\right|=n-a, \\
T^{F} \simeq \prod_{j}\left(\mathfrak{f}_{j}^{\times}\right)^{\lambda_{j}} \times\left(\mathfrak{f}_{2 j}^{1}\right)^{\lambda_{j}^{\prime},} & & |\lambda|+\left|\lambda^{\prime}\right|=n, \\
S^{F} \simeq \prod_{j}\left(\mathfrak{f}_{j}^{\times}\right)^{\mu_{j}} \times\left(\mathfrak{f}_{2 j}^{1}\right)^{\mu_{j}^{\prime}}, & |\mu|+\left|\mu^{\prime}\right|=n .
\end{array}
$$

We have $\left|j_{G_{\iota}}^{-1}(\operatorname{cl}(T, G))\right| \cdot\left|j_{H_{\iota}}^{-1}(\operatorname{cl}(S, H))\right|=1$ precisely when

$$
\nu_{j} \leq \lambda_{j}, \mu_{j} \quad \text { and } \quad \nu_{j}^{\prime} \leq \lambda_{j}^{\prime}, \mu_{j}^{\prime}
$$

for all $j$. We assume (9.4) holds from now on. Note that if $T$ and $S$ are anisotropic, then $\lambda_{j}=\mu_{j}=\nu_{j}=0$ for all $j$.

We count the number of $\iota$ in the sum (9.1) giving rise to a fixed pair of partitions $\left(\nu, \nu^{\prime}\right)$. For $s \in S_{\iota}^{F}$, consider the components $\left(s_{j 1}, \ldots, s_{j \mu_{j}} ; s_{j 1}^{\prime}, \ldots, s_{j \mu_{j}^{\prime}}^{\prime}\right)$ of $s$ in the $j^{\text {th }}$ block

$$
\left(\mathfrak{f}_{j}^{\times}\right)^{\mu_{j}} \times\left(\mathfrak{f}_{2 j}^{1}\right)^{\mu_{j}^{\prime}} \subset S^{F} .
$$

Then $\iota$ is determined by the pair of subsets

$$
\left\{k \in\left[1, \mu_{j}\right]: s_{j k}=1\right\}, \quad\left\{k^{\prime} \in\left[1, \mu_{j}^{\prime}\right]: s_{j k^{\prime}}^{\prime}=1\right\} .
$$

It follows that there are

$$
\left(\begin{array}{l}
\mu \\
\nu
\end{array}\right)\left(\begin{array}{l}
\mu^{\prime} \\
\nu^{\prime}
\end{array}\right)
$$

elements $\iota \in I(S)^{F}$ giving rise to $\left(\nu, \nu^{\prime}\right)$, where

$$
\left(\begin{array}{l}
\mu \\
\nu
\end{array}\right):=\prod_{j}\left(\begin{array}{c}
\mu_{j} \\
\nu_{j}
\end{array}\right), \quad\left(\begin{array}{l}
\mu^{\prime} \\
\nu^{\prime}
\end{array}\right):=\prod_{j}\left(\begin{array}{c}
\mu_{j}^{\prime} \\
\nu_{j}^{\prime}
\end{array}\right) .
$$

From equations (2.3) and $\left|j_{H_{\iota}}^{-1}(\operatorname{cl}(S, H))\right|=1$ we have

$$
\left|\bar{N}_{H}(\iota, S)^{F}\right|=\frac{\left|W_{H}(S)^{F}\right|}{\left|W_{H_{\iota}}(S)^{F}\right|}=\left(\begin{array}{c}
\mu \\
\nu
\end{array}\right)\left(\begin{array}{c}
\mu^{\prime} \\
\nu^{\prime}
\end{array}\right) \prod_{j}\left(\nu_{j} !\right)\left(\nu_{j}^{\prime} !\right)(2 j)^{\nu_{j}}(2 j)^{\nu_{j}^{\prime}} .
$$

Using Lemma 3.1 for $G_{\iota}$ and $H_{\iota}$, we find that

$$
(-1)^{\mathrm{rk} G_{\iota}+\mathrm{rk} H_{\iota}}=(-1)^{\mathrm{rk} G+\mathrm{rk} H+\sum \nu_{j}^{\prime}} .
$$

(One can also arrive at (9.6) by decomposing $U^{F}$ into irreducible $\mathfrak{f} Z_{\iota}^{F}$-modules, and calculating discriminants.)

Finally, we must calculate the pairing $\left\langle\chi_{v}, \eta_{\varsigma}\right\rangle_{Z_{\iota}^{F}}$. We may conjugate $T$ and $S$ to arrange that $Z_{\iota} \subset T \cap S$. Then

$$
\chi_{v}=\left.\frac{1}{\left|W_{G_{\iota}}(T)^{F}\right|} \sum_{x \in W_{G}(T)^{F}}\left({ }^{x} \chi\right)\right|_{Z_{\iota}}, \quad \eta_{\varsigma}=\left.\frac{1}{\left|W_{H_{\iota}}(S)^{F}\right|} \sum_{y \in W_{H}(S)^{F}}\left({ }^{y} \eta\right)\right|_{Z_{\iota}} .
$$

We now assume that $\chi$ and $\eta$ are regular, in the sense that they have trivial stabilizers in $W_{G}(T)^{F}$ and $W_{H}(S)^{F}$, respectively.

On the $j^{\text {th }}$ block $\left(\mathfrak{f}_{j}^{\times}\right)^{\mu_{j}} \times\left(\mathfrak{f}_{2 j}^{1}\right)^{\mu_{j}^{\prime}}$ of $S^{F}$, we have

$$
\eta=\eta_{j 1} \otimes \cdots \otimes \eta_{j \mu_{k}} \otimes \eta_{j 1}^{\prime} \otimes \cdots \otimes \eta_{j \mu_{k}^{\prime}}^{\prime} .
$$


Likewise, on the $j^{\text {th }}$ block $\left(\mathfrak{f}_{j}^{\times}\right)^{\lambda_{j}} \times\left(\mathfrak{f}_{2 j}^{1}\right)^{\lambda_{j}^{\prime}}$ of $T^{F}$, we have

$$
\chi=\chi_{j 1} \otimes \cdots \otimes \chi_{j \lambda_{j}} \otimes \chi_{j 1}^{\prime} \otimes \cdots \otimes \chi_{j \lambda_{j}^{\prime}}^{\prime}
$$

Define

$$
\begin{aligned}
& I_{j}=\left\{k \in\left[1, \mu_{j}\right]: \eta_{j k} \in \Gamma_{j} \cdot\left\{\chi_{j \ell}, \chi_{j \ell}{ }^{-1}\right\}, \quad \text { for some } \ell \in\left[1, \lambda_{j}\right]\right\}, \\
& I_{j}^{\prime}=\left\{k^{\prime} \in\left[1, \mu_{j}^{\prime}\right]: \eta_{j k^{\prime}}^{\prime}=\Gamma_{2 j} \cdot \chi_{j \ell}^{\prime}, \quad \text { for some } \ell \in\left[1, \lambda_{j}^{\prime}\right]\right\} .
\end{aligned}
$$

For every pair of subsets

$$
\left\{k_{1}, \cdots, k_{\nu_{j}}\right\} \subset I_{j}, \quad\left\{k_{1}^{\prime}, \cdots, k_{\nu_{j}^{\prime}}^{\prime}\right\} \subset I_{j}^{\prime},
$$

each of the

$$
\left(\nu_{j}\right) !\left(\nu_{j}^{\prime}\right) !(2 j)^{\nu_{j}}(2 j)^{\nu_{j}^{\prime}}
$$

conjugates of the character

$$
\eta_{j k_{1}} \otimes \cdots \otimes \eta_{j k_{\nu_{j}}} \otimes \eta_{j k_{1}^{\prime}}^{\prime} \otimes \cdots \otimes \eta_{j k_{\nu_{j}}^{\prime}}^{\prime}
$$

contributes exactly once to the pairing $\left\langle\chi_{v}, \eta_{\varsigma}\right\rangle_{Z_{\iota}^{F}}$, by the regularity assumption (1.3). It follows that

$$
\left\langle\chi_{v}, \eta_{\varsigma}\right\rangle_{Z_{\iota}^{F}}=\prod_{j}\left(\begin{array}{c}
\left|I_{j}\right| \\
\nu_{j}
\end{array}\right)\left(\begin{array}{c}
\left|I_{j}^{\prime}\right| \\
\nu_{j}^{\prime}
\end{array}\right)\left(\nu_{j}\right) !\left(\nu_{j}^{\prime}\right) !(2 j)^{\nu_{j}}(2 j)^{\nu_{j}^{\prime}} .
$$

Set

$$
e:=(-1)^{\mathrm{rk} G+\mathrm{rk} T+\mathrm{rk} H+\mathrm{rk} S} .
$$

Inserting (9.5), (9.6) and (9.8) into (9.1), and summing over all $\left(\nu, \nu^{\prime}\right)$ satisfying (9.4), we get

$$
\begin{aligned}
e \cdot\left\langle R_{T, \chi}^{G}, R_{S, \eta}^{H}\right\rangle_{H^{F}} & =\sum_{\nu, \nu^{\prime}}(-1)^{\sum \nu_{j}^{\prime}} \prod_{j}\left(\begin{array}{c}
\left|I_{j}\right| \\
\nu_{j}
\end{array}\right)\left(\begin{array}{c}
\left|I_{j}^{\prime}\right| \\
\nu_{j}^{\prime}
\end{array}\right) \\
& =\prod_{j}\left[\left(\sum_{\nu_{j}=0}^{\left|I_{j}\right|}\left(\begin{array}{c}
\left|I_{j}\right| \\
\nu_{j}
\end{array}\right)\right) \cdot\left(\sum_{\nu_{j}^{\prime}=0}^{\left|I_{j}^{\prime}\right|}(-1)^{\nu_{j}^{\prime}}\left(\begin{array}{c}
\left|I_{j}^{\prime}\right| \\
\nu_{j}^{\prime}
\end{array}\right)\right)\right] \\
& = \begin{cases}2^{r} & \text { if } I_{j}^{\prime} \text { is empty for all } j, \\
0 & \text { otherwise, }\end{cases}
\end{aligned}
$$

where

$$
r=\sum_{j}\left|I_{j}\right| .
$$

If either $T$ or $S$ is anisotropic, then $r=0$. This proves Theorem 1.2 .

\section{Restriction From $\mathrm{SO}_{7}$ TO $G_{2}$}

The previous situation had $\delta=0$. We now consider a case where $\delta=1$. The simplest such case is $G=G_{2}, H=S L_{3}$, which we leave to the reader.

Here we take $G=S O_{7}, H=G_{2}$, embedded in $G$ via the irreducible 7-dimensional representation $V$ of $G_{2}$. We have

$$
\delta=9-8=1 .
$$

We assume $p \geq 5$. 
We will calculate the multiplicities $\left\langle R_{T, \chi}^{G}, R_{S, \eta}^{H}\right\rangle_{H^{F}}$, using the formula of section 6.3. We do not need any detailed knowledge of Green functions, beyond the general facts about their degrees and leading terms that we have already used.

Let $\alpha, \beta$ be simple roots of a maximal f-split torus $S_{0}$ in $H$, with $\alpha$ short. The nonzero weights of $H$ in $V$ are the short roots of $S_{0}$. We view the maximal f-split tori $T_{0}$ and $S_{0}$ as

$$
T_{0}=\left\{(x, y, z) \in \mathfrak{F}^{3}: x y z \neq 0\right\}, \quad S_{0}=\left\{(x, y, z) \in \mathfrak{F}^{3}: x y z=1\right\},
$$

in such a way that the coordinate functions $e_{1}, e_{2}, e_{3}$ on $T_{0}$ restrict to the roots $2 \alpha+\beta,-\alpha,-\alpha-\beta$ on $S_{0}$. In this realization, the simple co-roots of $S_{0}$ in $H$ are

$$
\check{\alpha}(t)=\left(t, t^{-2}, t\right), \quad \check{\beta}(t)=\left(1, t, t^{-1}\right),
$$

and the corresponding simple reflections $r_{\alpha}, r_{\beta}$ in the Weyl group $W_{H}$ act by

$$
r_{\alpha} \cdot\left(t_{1}, t_{2}, t_{3}\right)=\left(t_{3}^{-1}, t_{2}^{-1}, t_{1}^{-1}\right), \quad r_{\beta} \cdot\left(t_{1}, t_{2}, t_{3}\right)=\left(t_{1}, t_{3}, t_{2}\right) .
$$

Since $S_{0}$ contains regular elements in $T_{0}$, it follows that $W_{H}$ is a subgroup of $W_{G}$. If $W_{G}$ is realized as the group of the cube, then $W_{H}$ is the subgroup preserving a diagonal of the cube; as coset representatives for $W_{G} / W_{H}$ we may take the identity and each coordinate sign change.

Let $T, S$ be $F$-stable maximal tori in $G$ and $H$, corresponding to the conjugacy classes of $x \in W_{G}$ and $y \in W_{H}$, respectively. Let $\chi \in \operatorname{Irr}\left(T^{F}\right), \eta \in \operatorname{Irr}\left(S^{F}\right)$.

We will use the refined multiplicity formula of section 6.3 . We first tabulate the pairs $(\iota, u)$ in $H$, with $\iota \in I\left(S_{0}\right)$ and $u \in H_{\iota}$, for which

$$
\operatorname{dim} Z_{\iota}+d_{G_{\iota}}(u)+d_{H_{\iota}}(u)-\operatorname{dim} C_{H_{\iota}}(u) \geq 0 .
$$

We find four types as shown:

\begin{tabular}{|c|c|c|}
\hline type & $\iota$ & $u$ \\
\hline \hline$a$ & $(1,1,1)$ & $1, u_{0}$ \\
\hline$b$ & $\left(1, t, t^{-1}\right), t^{q}=t \neq \pm 1$ & 1 \\
\hline$c$ & $\left(1, t, t^{-1}\right), t^{q}=t^{-1} \neq \pm 1$ & 1 \\
\hline$d$ & regular & 1 \\
\hline
\end{tabular}

The middle column shows a typical element in $S_{0}$ for each type of $\iota$. There can be more than one $\iota$ of the same type. Here $u_{0} \in H^{F}$ is a long root element, which has Jordan partition $1^{3} 2^{2}$ on $V$.

From equation (5.18), we have

$$
M(q)=M_{a}(q)+M_{b}(q)+M_{c}(q)+M_{d}(q),
$$

where each term on the right is the sum

$$
\sum \Psi_{\alpha} \Theta_{\alpha}
$$

over those $\alpha$ whose $\iota$ component is $H$-conjugate to an $\iota$ of the corresponding type $a, b, c, d$ in the table above. Taking the polynomial part of each sum, as in section 6.3. we have

$$
M(q)=P_{a}(q)+P_{b}(q)+P_{c}(q)+P_{d}(q) .
$$

We now calculate each of the four terms on the right side of (10.2).

Type a: The maps $j_{G_{\iota}}$ and $j_{H_{\iota}}$ are the identity. The centralizers of $u_{0}$ in $G$ and $H$ are both connected. Let $\phi_{G}, \phi_{H}$ be the Springer representations of $W_{G}$ and $W_{H}$ corresponding to $u_{0}$, let $\rho_{G}, \rho_{H}$ be the reflection representations, and let $\epsilon_{G}$, $\epsilon_{H}$ be the sign representations. 
From [5, 13.3], we find that $\phi_{G}$ is the unique two-dimensional representation of $W_{G}=S_{3} \ltimes\{ \pm 1\}^{3}$ which is irreducible on $S_{3}$ and nontrivial on $\{ \pm 1\}^{3}$, and $\phi_{H}$ is the one-dimensional character of $W_{H}$ given by $\phi_{H}\left(r_{\alpha}\right)=-1, \phi_{H}\left(r_{\beta}\right)=+1$.

In the following calculation we write $R_{1} \sim R_{2}$ for rational functions $R_{i}$ such that $\operatorname{deg}\left(R_{1}-R_{2}\right)<0$. We have

$$
M_{a}(q)=\frac{Q_{T}^{G}(1) Q_{S}^{H}(1)}{\left|H^{F}\right|}+\frac{Q_{T}^{G}\left(u_{0}\right) Q_{S}^{H}\left(u_{0}\right)}{\left|C_{H}\left(u_{0}\right)^{F}\right|} .
$$

The $u_{0}$ term has degree zero, so we may replace it by its leading term:

$$
M_{a}(q) \sim \frac{Q_{T}^{G}(1) Q_{S}^{H}(1)}{\left|H^{F}\right|}+\phi_{G}(x) \phi_{H}(y) .
$$

Since

$$
\begin{aligned}
& \left|T^{F}\right|=\operatorname{det}(q-x)=q^{3}-\rho_{G}(x) q^{2}+\cdots, \\
& \left|S^{F}\right|=\operatorname{det}(q-y)=q^{2}-\rho_{H}(y) q+\cdots,
\end{aligned}
$$

it follows that

$$
\begin{aligned}
\epsilon_{G}(x) \epsilon_{H}(y) \frac{Q_{T}^{G}(1) Q_{S}^{H}(1)}{\left|H^{F}\right|} & =\frac{\left[G^{F}: T^{F}\right]_{p^{\prime}} \cdot\left[H^{F}: S^{F}\right]_{p^{\prime}}}{\left|H^{F}\right|} \\
& =\frac{\left(q^{6}-1\right)\left(q^{4}-1\right)\left(q^{2}-1\right)}{q^{6}\left|T^{F}\right|\left|S^{F}\right|} \\
& \sim q+\rho_{G}(x)+\rho_{H}(y) .
\end{aligned}
$$

We get

$$
P_{a}(q)=\epsilon_{G}(x) \epsilon_{H}(y)\left[q+\rho_{G}(x)+\rho_{H}(y)\right]+\phi_{G}(x) \phi_{H}(y) .
$$

Type b: For $\iota \in I(S)^{F}$ of type $b$, the elements of $S_{\iota}^{F}$ are $H$-conjugate to elements of the form $s=\left(1, t, t^{-1}\right) \in S_{0}$ for some $t \in \mathfrak{f}^{\times}, t^{2} \neq 1$. We have

$$
G_{\iota}=S O_{3} \times G L_{2}, \quad H_{\iota}=G L_{2} .
$$

The stabilizer $W_{H_{\iota}}^{\prime}$ of $s$ in $W_{H}$ is generated by the reflection

$$
r_{0}:=r_{\alpha} r_{\beta} r_{\alpha} r_{\beta} r_{\alpha} \in W_{H} .
$$

The number of $\iota \in I(S)^{F}$ of type $b$ is given by

$$
\nu_{b}(y):= \begin{cases}3 & \text { if } y=1, \\ 1 & \text { if }[y]=\left[r_{0}\right] \\ 0 & \text { otherwise }\end{cases}
$$

where [.] denotes a conjugacy class in $W_{H}$. We have

for $y \in\left\{1, r_{0}\right\}$.

$$
\frac{\nu_{b}(y)}{\left|\bar{N}_{H}(\iota, S)^{F}\right|}=\nu_{b}(y) \cdot \frac{\left|C_{W_{H_{\iota}}^{\prime}}(y)\right|}{\left|C_{W_{H}}(y)\right|}=\frac{1}{2}
$$

The roots of $T_{0}$ vanishing on $s$ are $e_{1}, e_{2}+e_{3}$. The corresponding reflections $r_{1}, r_{2} \in W_{G}$ generate the stabilizer $W_{G_{\iota}}^{\prime}$ of $s$ in $W_{G}$. For $s \in S_{\iota}^{F}$, the element $y_{s}$ of section 2 is $y_{s}=1$. Hence, the mapping

$$
j_{G_{\iota}}: H^{1}\left(F, W_{G_{\iota}}^{\prime}\right) \rightarrow H^{1}\left(F, W_{G}\right)
$$

of (2.2) is induced by the inclusion $W_{G_{\iota}}^{\prime} \hookrightarrow W_{G}$; its image consists of the four classes in $W_{G}$ represented by elements in $W_{G_{\iota}}^{\prime}$, as shown in the following table: 


\begin{tabular}{|c|c|c|c|c|}
\hline$H^{1}\left(F, W_{G_{\iota}}^{\prime}\right)$ & 1 & $r_{1}$ & $r_{2}$ & $r_{1} r_{2}$ \\
\hline$H^{1}\left(F, W_{G}\right)$ & {$\left[1^{3},-\right]$} & {$\left[1^{2}, 1\right]$} & {$[12,-]$} & {$[2,1]$} \\
\hline
\end{tabular}

Set

$$
W_{b}:=W_{G_{\iota}}^{\prime} \times W_{H_{\iota}}^{\prime} .
$$

Using section 7 and taking (10.4) into account, it follows that

$$
P_{b}(q)=\frac{1}{2} \epsilon_{G}(x) \epsilon_{H}(y)\left\langle\chi_{x}, \eta_{y}\right\rangle_{Z_{\iota}^{F}}
$$

if $(x, y)$ is $\left(W_{G} \times W_{H}\right)$-conjugate to an element of $W_{b}$, and $P_{b}(q)=0$ otherwise. Here we have written $\chi_{x}$ instead of $\chi_{v}$, where $\{v\}=j_{G_{\iota}}^{-1}([x])$, and likewise for $\eta_{y}$.

Type c: For $\iota \in I(S)^{F}$ of type $c$, the elements of $S_{\iota}^{F}$ are $H$-conjugate to elements of the form $s=\left(1, t, t^{-1}\right) \in S_{0}$ for some $t \in \mathfrak{f}_{2}^{1}, t^{2} \neq 1$. We have

$$
G_{\iota}=S_{3} \times U_{2}, \quad H_{\iota}=U_{2} .
$$

The groups

$$
W_{G_{\iota}}^{\prime}=\left\langle r_{1}, r_{2}\right\rangle, \quad W_{H_{\iota}}^{\prime}=\left\langle r_{0}\right\rangle
$$

are as in type $b$. The number of $\iota \in I(S)^{F}$ of type $c$ is given by

$$
\nu_{c}(y):= \begin{cases}3 & \text { if } y=-1, \\ 1 & \text { if }[y]=\left[-r_{0}\right] \\ 0 & \text { otherwise. }\end{cases}
$$

As before, we have

$$
\frac{\nu_{c}(y)}{\left|\bar{N}_{H}(\iota, S)^{F}\right|}=\nu_{c}(y) \cdot \frac{\left|C_{W_{H_{\iota}}^{\prime}}(y)\right|}{\left|C_{W_{H}}(y)\right|}=\frac{1}{2},
$$

now for $y \in\left\{-1,-r_{0}\right\}$.

Let $r$ be the reflection about $e_{2}-e_{3}$. For $s \in S_{\iota}^{F}$, the element $y_{s}$ of section 2 is $y_{s}=r$. Hence, the mapping

$$
j_{G_{\iota}}: H^{1}\left(F, W_{G_{\iota}}^{\prime}\right) \rightarrow H^{1}\left(F, W_{G}\right)
$$

is induced by the map $x \mapsto x r$, as shown in the following table:

\begin{tabular}{|c|c|c|c|c|}
\hline$H^{1}\left(F, W_{G_{\iota}}^{\prime}\right)$ & 1 & $r_{1}$ & $r_{2}$ & $r_{1} r_{2}$ \\
\hline$H^{1}\left(F, W_{G}\right)$ & {$[12,-]$} & {$[2,1]$} & {$\left[1,1^{2}\right]$} & {$\left[-, 1^{3}\right]$} \\
\hline
\end{tabular}

Set

$$
W_{c}:=W_{G_{\iota}}^{\prime} r \times W_{H_{\iota}}^{\prime} r .
$$

Using section 7 and taking (10.6) into account, it follows that

$$
P_{c}(q)=\frac{1}{2} \epsilon_{G}(x) \epsilon_{H}(y)\left\langle\chi_{x}, \eta_{y}\right\rangle_{Z_{\iota}^{F}}
$$

if $(x, y)$ is $\left(W_{G} \times W_{H}\right)$-conjugate to an element of $W_{c}$, and $P_{c}(q)=0$ otherwise. Again, we have written $\chi_{x}$ instead of $\chi_{v}$, where $\{v\}=j_{G_{\iota}}^{-1}([x])$, and likewise for $\eta_{y}$.

Type d: In this case $S_{\iota}$ contains regular elements in $G$, so $H_{\iota}=Z_{\iota}=S$, and $G_{\iota}=C_{G}(S)$ is a maximal torus in $G$. Hence $P_{d}(q) \neq 0$ only if $T$ is $G^{F}$-conjugate to $C_{G}(S)$. The mapping $S \mapsto C_{G}(S)$ is given, in terms of conjugacy classes in $W_{H}$ and $W_{G}$, in the following table:

\begin{tabular}{|c|c|c|c|c|c|c|}
\hline$S$ & 1 & $r_{\alpha}$ & $r_{\beta}$ & -1 & $\left(r_{\alpha} r_{\beta}\right)^{2}$ & $r_{\alpha} r_{\beta}$ \\
\hline$C_{G}(S)$ & {$\left[1^{3},-\right]$} & {$[2,1]$} & {$[12,-]$} & {$\left[-, 1^{3}\right]$} & {$[3,-]$} & {$[-, 3]$} \\
\hline
\end{tabular}


Denote the embedding

$$
W_{H} \hookrightarrow W_{G} \quad \text { by } y \mapsto \tilde{y} .
$$

Let

$$
W_{d}=\left\{(\tilde{y}, y): y \in W_{H}\right\} \subset W_{G} \times W_{H} .
$$

For $(x, y) \in W_{d}$, we may assume that $T=C_{G}(S)$. Then

$$
\Theta_{\alpha}(q) \sim \sum_{\substack{w \in W_{G}(T)^{F} \\ v \in W_{H}(S)^{F}}}\left\langle{ }^{w} \chi,{ }^{v} \eta\right\rangle_{S^{F}}=\left|W_{H}(S)^{F}\right| \sum_{w \in W_{G}(T)^{F}}\left\langle{ }^{w} \chi, \eta\right\rangle_{S^{F}} .
$$

Since

it follows that

$$
\Psi_{\alpha}(t)=\frac{1}{\left|W_{H}(S)^{F}\right|},
$$

$$
P_{d}(q)=\sum_{w \in W_{G}(T)^{F}}\left\langle{ }^{w} \chi, \eta\right\rangle_{S^{F}}=\left\langle\chi_{x}, \eta\right\rangle_{S^{F}}
$$

if $(x, y)$ is $\left(W_{G} \times W_{H}\right)$-conjugate to an element of $W_{d}$, and $P_{d}(q)=0$ otherwise.

10.1. Cuspidal multiplicities. From now on we assume that our tori $T, S$ are anisotropic, and that the characters $\chi$ and $\eta$ are regular. We will make the multiplicities computed above more precise.

The elliptic classes in $W\left(B_{n}\right)$ are those of the form $[-, \lambda]$, where $\lambda$ is a partition of $n$. So in $W_{G}$ we have three elliptic classes:

$$
[-, 3], \quad[-, 12], \quad\left[-, 1^{3}\right] .
$$

The first of these is the Coxeter class and the last is $\{-1\}$.

In $W_{H}$ we also have three elliptic classes, represented by the powers

$$
\text { cox, } \quad \operatorname{cox}^{2}, \quad \operatorname{cox}^{3}=-1
$$

of a Coxeter element cox $=r_{\alpha} r_{\beta}$. Via the embedding $W_{H} \hookrightarrow W_{G}$, the elements -1 , cox of $W_{H}$ are also the -1 and Coxeter elements of $W_{G}$.

Let $x \in \operatorname{cl}(T, G), y \in \operatorname{cl}(S, H)$. Note that $P_{b}(q)=0$. Combining formulas (10.3), (10.7), (10.8), we get the multiplicity formula

$$
-\left\langle R_{T, \chi}^{G}, R_{S, \eta}^{H}\right\rangle_{H^{F}}=q+\rho_{G}(x)+\rho_{H}(y)-\phi_{G}(x) \phi_{H}(y)+a(x, y)
$$

where

$$
a(x, y)= \begin{cases}\frac{1}{2}\left\langle\chi_{-1}, \eta_{-1}\right\rangle_{Z_{c}^{F}}-\left\langle\chi_{-1}, \eta\right\rangle_{Z_{d}^{F}} & \text { if } x=y=-1 \\ -\left\langle\chi_{\mathrm{cox}}, \eta\right\rangle_{Z_{d}^{F}} & \text { if } x=y=\mathrm{cox} \\ 0 & \text { otherwise. }\end{cases}
$$

Here we have written $Z_{c}$ for $Z_{\iota}$, when $\iota$ has type $c$, and likewise for $Z_{d}=S$. The numbers

$$
\left\langle\chi_{-1}, \eta_{-1}\right\rangle_{Z_{c}^{F}}, \quad\left\langle\chi_{-1}, \eta_{-1}\right\rangle_{Z_{d}^{F}}, \quad\left\langle\chi_{\mathrm{cox}}, \eta\right\rangle_{Z_{d}^{F}}
$$

are calculated explicitly in (10.11), (10.13) and (10.16) below.

We calculate $\rho_{G}(x)+\rho_{H}(y)-\phi_{G}(x) \phi_{H}(y)$ from the character tables of $W_{G}$ and $W_{H}$ and then set $A(x)=a(x, x)$ for $x \in\{-1, \operatorname{cox}\}$ to arrive at the multiplicities in Table 1 .

Note that $S_{-1}$ has regular characters only for $q \geq 5$ (which we have already assumed), and $T_{-1}$ has regular characters only for $q \geq 7$. For $q=5$ we get Table 2 , 


\begin{tabular}{|c|c|c|c|}
\hline$y \backslash x$ & {$\left[-, 1^{3}\right]=[-1]$} & {$[-, 12]$} & {$[-, 3]=[\operatorname{cox}]$} \\
\hline \hline$\left[\operatorname{cox}^{3}\right]=[-1]$ & $q-7+A(-1)$ & $q-3$ & $q-1$ \\
\hline$\left[\operatorname{cox}^{2}\right]$ & $q-2$ & $q-2$ & $q-2$ \\
\hline$\left[\operatorname{cox}^{2}\right]$ & $q-4$ & $q$ & $q+2-A(\operatorname{cox})$ \\
\hline
\end{tabular}

TABLE 1. $-\left\langle R_{T_{x}, \chi}^{G}, R_{S_{y}, \eta}^{H}\right\rangle_{H^{F}}$ for $q \geq 7$

\begin{tabular}{|c|c|c|c|}
\hline$y \backslash x$ & {$\left[-, 1^{3}\right]=[-1]$} & {$[-, 12]$} & {$[-, 3]=[\operatorname{cox}]$} \\
\hline \hline$\left[\cos ^{3}\right]=[-1]$ & - & 2 & 4 \\
\hline$\left[\operatorname{cox}^{2}\right]$ & - & 3 & 3 \\
\hline$[\operatorname{cox}]$ & - & 5 & 6 or 7 \\
\hline
\end{tabular}

TABLE 2. $-\left\langle R_{T_{x}, \chi}^{G}, R_{S_{y}, \eta}^{H}\right\rangle_{H^{F}}$ for $q=5$

where the dichotomy in the (cox, cox) entry arises from the fact that $A(\operatorname{cox})=0$ or 1, depending on $\chi$ and $\eta$ (see below).

The rest of this section is devoted to the explicit calculation of $A(-1)$ and $A($ cox $)$.

A(-1): We identify

$$
\begin{gathered}
T_{-1}^{F}=\left(\mathfrak{f}_{2}^{1}\right)^{3}, \quad S_{-1}^{F}=\left\{(x, y, z) \in T_{-1}^{F}: x y z=1\right\}, \\
\chi=\chi_{1} \otimes \chi_{2} \otimes \chi_{3}, \quad \eta=\operatorname{Res}_{S_{-1}^{F}}\left(\eta_{1} \otimes \eta_{2} \otimes \eta_{3}\right), \quad \text { with } \quad \chi_{i}, \eta_{i} \in \operatorname{Irr}\left(\mathfrak{f}_{2}^{1}\right) .
\end{gathered}
$$

Recall that

$$
A(-1)=\frac{1}{2}\left\langle\chi_{-1}, \eta_{-1}\right\rangle_{Z_{c}}-\left\langle\chi_{-1}, \eta\right\rangle_{Z_{d}} .
$$

We have $Z_{c}^{F}=\left\{\left(1, t, t^{-1}\right): t \in \mathfrak{f}_{2}^{1}\right\}$ and

$$
\left\langle\chi_{-1}, \eta_{-1}\right\rangle_{Z_{c}^{F}}=2 \sum_{i<j}\left\langle\frac{\chi_{i}}{\chi_{j}}+\frac{\chi_{j}}{\chi_{i}}+\chi_{i} \chi_{j}+\frac{1}{\chi_{i} \chi_{j}}, \frac{\eta_{1}}{\eta_{2}}+\frac{\eta_{2}}{\eta_{3}}+\frac{\eta_{3}}{\eta_{1}}\right\rangle_{\mathrm{f}_{2}^{1}} .
$$

For $\iota$ of type $d$, we have $Z_{d}=S_{-1}$. The $W_{G}$-orbit of $\chi$ breaks up into four $W_{H}$-orbits:

$$
\begin{aligned}
& \tilde{\mathcal{O}}_{0}=W_{H} \cdot\left(\chi_{1} \otimes \chi_{2} \otimes \chi_{3}\right), \\
& \tilde{\mathcal{O}}_{1}=W_{H} \cdot\left(\bar{\chi}_{1} \otimes \chi_{2} \otimes \chi_{3}\right), \\
& \tilde{\mathcal{O}}_{2}=W_{H} \cdot\left(\chi_{1} \otimes \bar{\chi}_{2} \otimes \chi_{3}\right), \\
& \tilde{\mathcal{O}}_{3}=W_{H} \cdot\left(\chi_{1} \otimes \chi_{2} \otimes \bar{\chi}_{3}\right) .
\end{aligned}
$$

These restrict to $W_{H^{-}}$orbits $\mathcal{O}_{0}, \ldots, \mathcal{O}_{3}$ in $\operatorname{Irr}\left(S_{-1}^{F}\right)$. Even though the orbits $\tilde{\mathcal{O}}_{i}$ consist of $W_{G}$-regular characters, the characters in $\mathcal{O}_{i}$ need not be $W_{H}$-regular. Moreover, it can happen that $\mathcal{O}_{i}=\mathcal{O}_{j}$ for $i \neq j$. In any case, formula (10.8) gives

$$
\left\langle\chi_{-1}, \eta\right\rangle_{Z_{d}^{F}}=\left|\left\{i \in[0,3]: \eta \in \mathcal{O}_{i}\right\}\right| .
$$

We illustrate with $q=7$. The unique regular $W_{G}$-orbit in $\operatorname{Irr}\left(T_{-1}^{F}\right)$ contains the character $\chi=\zeta \otimes \zeta^{2} \otimes \zeta^{3}$, where $\zeta$ is a faithful character of $\mathfrak{f}_{2}^{1} \simeq \mu_{8}$. There are two $W_{H}$-orbits of regular characters $\eta, \eta^{\prime}$ in $\operatorname{Irr}\left(S_{-1}^{F}\right)$, distinguished as follows: $\eta$ 
belongs to the restrictions $\mathcal{O}_{1}=\mathcal{O}_{3}$, and $\eta^{\prime}=\operatorname{Res}_{S_{-1}^{F}}\left(\zeta \otimes 1 \otimes \zeta^{-2}\right)$ does not extend to a regular character of $T_{-1}^{F}$. Formulas (10.11) and (10.13) give

$$
\begin{array}{ll}
\left\langle\chi_{-1}, \eta_{-1}\right\rangle_{Z_{c}^{F}}=12, & \left\langle\chi_{-1}, \eta_{-1}\right\rangle_{Z_{d}^{F}}=2, \\
\left\langle\chi_{-1}, \eta_{-1}^{\prime}\right\rangle_{Z_{c}^{F}}=10, & \left\langle\chi_{-1}, \eta_{-1}^{\prime}\right\rangle_{Z_{d}^{F}}=0 .
\end{array}
$$

From Table 1 we get

$$
\begin{aligned}
-\left\langle R_{T_{-1}, \chi}^{G}, R_{S_{-1}, \eta}^{H}\right\rangle_{H^{F}} & =7-7+\frac{1}{2} \cdot 12-2=4, \\
-\left\langle R_{T_{-1}, \chi}^{G}, R_{S_{-1}, \eta^{\prime}}^{H}\right\rangle_{H^{F}} & =7-7+\frac{1}{2} \cdot 10-0=5 .
\end{aligned}
$$

$\mathbf{A}(\mathbf{c o x})$ : Here the only relevant type is $d$. We identify

$$
T_{\text {cox }}^{F}=\mathfrak{f}_{6}^{1}=\operatorname{ker}\left[\mathfrak{f}_{6}^{\times} \stackrel{\text { norm }}{\longrightarrow} \mathfrak{f}_{3}^{\times}\right], \quad S_{\text {cox }}^{F}=\left(\mathfrak{f}_{6}^{1}\right)^{q+1},
$$

$W\left(T_{\text {cox }}\right)^{F}=W\left(S_{\text {cox }}\right)^{F}$ is cyclic of order six, and acts on $T_{\text {cox }}^{F}$ and $S_{\text {cox }}^{F} \operatorname{via} \operatorname{Gal}\left(\mathfrak{f}_{6} / \mathfrak{f}\right)$. If $\chi \in \operatorname{Irr}\left(T_{\text {cox }}^{F}\right)$ and $\eta \in \operatorname{Irr}\left(S_{\text {cox }}^{F}\right)$ are both regular, and $\eta$ appears in the restriction of some Galois conjugate of $\chi$, then the restriction map $\operatorname{Irr}\left(T_{\text {cox }}^{F}\right) \longrightarrow \operatorname{Irr}\left(S_{\text {cox }}^{F}\right)$ maps $W_{G} \cdot \chi$ bijectively onto $W_{H} \cdot \eta$. Formula (10.8) gives

$$
A(\operatorname{cox})=\left\langle\chi_{\text {cox }}, \eta\right\rangle_{Z_{d}^{F}}= \begin{cases}1 & \text { if } \eta=\operatorname{Res}\left({ }^{w} \chi\right) \text { for some } w \in \operatorname{Gal}\left(\mathfrak{f}_{6} / \mathfrak{f}\right), \\ 0 & \text { otherwise. }\end{cases}
$$

Hence the (cox, cox) entry in Table 1 is made precise:

$$
-\left\langle R_{T_{\mathrm{cox}}, \chi}^{G}, R_{S_{\mathrm{cox}}, \eta}^{H}\right\rangle_{H^{F}}= \begin{cases}q+1 & \text { if } \eta=\operatorname{Res}\left({ }^{w} \chi\right) \text { for some } w \in \operatorname{Gal}\left(\mathfrak{f}_{6} / \mathfrak{f}\right), \\ q+2 & \text { otherwise. }\end{cases}
$$

\section{REFERENCES}

[1] D. Akhiezer and D. Panyushev, Multiplicities in the branching rules and the complexity of homogeneous spaces, Moscow Math. Jour., 2 no. 1, (2002) pp. 17-33. MR1900582 (2003d:14057)

[2] E. Bannai, N. Kawanaka, and S.Y. Song, The character table of the Hecke algebra $\mathcal{H}\left(G L_{2 n}\left(\mathbf{F}_{q}\right), S p_{2 n}\left(\mathbf{F}_{q}\right)\right)$, J. Algebra, 129 (1990), no. 2, pp. 320-366. MR.1040942(91d:20052)

[3] M. Beynon and N. Spaltenstein, Tables of Green Polynomials for exceptional groups, Warwick computer science centre report no. 23 (1986).

[4] A. Borel, Linear algebraic groups, second enlarged edition, Graduate Texts in Mathematics, vol. 126, Springer-Verlag, New York, 1991. MR1102012 (92d:20001)

[5] R. Carter, Finite groups of Lie type. Conjugacy classes and complex characters, John Wiley \& Sons, Ltd., Chichester, 1993. MR1266626 (94k:20020)

[6] _ Semisimple conjugacy classes and classes in the Weyl group, Jour. of Alg., 260 (2003) pp. 99-110. MR 1973577 (2004b:20071)

[7] S. DeBacker and M. Reeder, Depth-Zero Supercuspidal L-packets and their Stability, preprint 2004.

[8] P. Deligne and G. Lusztig, Representations of reductive groups over finite fields, Ann. of Math., 103 (1976) pp. 103-161. MR0393266 (52:14076)

[9] M. Geck, G. Hiss, F. Lübeck, G. Malle, and G. Pfeiffer, CHEVIE - A system for computing and processing generic character tables for finite groups of Lie type, Weyl groups and Hecke algebras, Appl. Algebra Engrg. Comm. Comput., 7 (1996) pp. 175-210. MR.1486215 (99m:20017)

[10] B. Gross, On the centralizer of a regular, semi-simple, stable conjugacy class, Electronic J. of Representation Theory (2005). MR2133761 (2006a:20089)

[11] B. Gross and D. Prasad, On the decomposition of a representation of $\mathrm{SO}_{n}$ when restricted to $S O_{n-1}$, Canad. J. Math., 44 (1992), pp. 974-1002. MR.1186476 (93j:22031)

[12] B. Gross and M. Reeder, From Laplace to Langlands via representations of orthogonal groups, Bull. Amer. Math. Soc., 43 (2006), pp. 163-205. 
[13] T. Hagedorn, Multiplicities in Restricted Representations of $G L_{n}\left(\mathbf{F}_{q}\right), U_{n}\left(\mathbf{F}_{q^{2}}\right), S O_{n}\left(\mathbf{F}_{q}\right)$, Ph.D. thesis, Harvard University (1994).

[14] D. Kazhdan, Proof of Springer's hypothesis, Israel J. Math., 28 (1977), pp. 272-286. MR0486181 (58:5959)

[15] D. Luna, Sur les orbites fermées des groupes algébriques réductifs, Invent. Math., 16 (1972), pp. 1-5. MR0294351 (45:3421)

[16] G. Lusztig, Green functions and character sheaves, Ann. Math., 131 (1990), pp. 355-408. MR1043271 (91c:20054)

[17] _ Symmetric spaces over a finite field, Grothendieck Festschrift, III Birkhäuser(1990), pp. 57-81. MR.1106911 (92e:20034)

[18] T. Shoji, On the Green polynomials of a Chevalley group of type $F_{4}$, Comm. in Algebra, 10 (1982), pp. 505-543. MR0647835 (83d:20030)

[19] - On the Green polynomials of classical groups, Invent. Math., 74 (1983), pp. 239-267. MR0723216 (85f:20032)

[20] , Green functions of reductive groups over a finite field, Proc. Symp. Pure Math., (47), Amer. Math. Soc. (1987), pp. 289-301. MR0933366 (88m:20014)

[21] T. A. Springer, Trigonometric sums, Green functions of finite groups and representations of Weyl groups, Invent. Math., 36 (1976), pp. 173-207. MR0442103 (56:491)

[22] _ A construction of representations of Weyl groups, Invent. Math., 44 (1978), pp. 279293. MR0491988 (58:11154)

[23] __ A purity result for fixed point varieties in flag manifolds, J. Fac. Sci. Univ. Tokyo Sect. IA Math., 31 (1984), pp. 271-282. MR0763421 (86c:14034)

[24] T. A. Springer and R. Steinberg, Conjugacy Classes, Seminar in algebraic groups and related finite groups, Lecture Notes in Math., 131 (1970), pp. 167-266. MR0268192 (42:3091)

[25] R. Steinberg, On the desingularization of the unipotent variety, Invent. Math., 36 (1976), pp. 209-224. MR0430094 (55:3101)

[26] B. Srinivasan, Green polynomials for finite classical groups, Comm. in Algebra, 5 (1976), pp. 1241-1259. MR0498889(58:16905)

[27] E. Thoma, Die Einschränkung der Charaktere von $G L(n, q)$ auf $G L(n-1, q)$, Math. Z., 119 (1971), pp. 321-338. MR0288190 (44:5388)

Department of Mathematics, Boston College, Chestnut Hill, Massachusetts 02467

E-mail address: reederma@bc.edu 